\title{
ON AN ITERATIVE METHOD FOR APPROXIMATE SOLUTIONS OF A GENERALIZED BOUSSINESQ MODEL
}

\author{
JOSÉ LUIZ BOLDRINI, BLANCA CLIMENT-EZQUERRA, \\ MARÍA DRINA ROJAS-MEDAR, AND MARKO A. ROJAS-MEDAR
}

\begin{abstract}
An iterative method is proposed for finding approximate solutions of an initial and boundary value problem for a nonstationary generalized Boussinesq model for thermally driven convection of fluids with temperature dependent viscosity and thermal conductivity. Under certain conditions, it is proved that such approximate solutions converge to a solution of the original problem; moreover, convergence-rate bounds for the constructed approximate solutions are also obtained.
\end{abstract}

\section{INTRODUCTION}

Boussinesq type systems of hydrodynamic equations arise as zero order approximations to the coupling between the Navier-Stokes equation and the thermodynamic equation (see Joseph [15], Chandrasekhar [2], Feireisl [6], Rajagopal, Ruzicka and Srinivasa [25]). In the derivations of such systems, it is usual to assume that the fluid viscosity and thermal conductivity are positive constants; however, there are several important physical situations where such hypotheses are not adequate, and one must consider the possibility that such viscosity and thermal conductivity may be temperature dependent. The experiments done by von Tippelkirch [31], for instance, clearly confirm the influence of the viscosity dependent temperature on the main macroscopic features of the flow, and thus the necessity of analyzing such more complex situations. A mathematical model for this case (see Drazin and Reid [4]) is the following: given $\Omega \subset \mathbb{R}^{N}(N=2$ ou 3) and $T>0$ find the field $\mathbf{u}: \Omega \times(0, T) \rightarrow \mathbb{R}^{N}$ and scalar functions $\varphi, p: \Omega \times(0, T) \rightarrow \mathbb{R}$ satisfying the system of equations:

$$
\begin{array}{lr}
\frac{\partial \mathbf{u}}{\partial t}+\mathbf{u} \cdot \nabla \mathbf{u}-\operatorname{div}(\nu(\varphi) \nabla \mathbf{u})+\nabla p=\alpha \varphi \mathbf{g}+\mathbf{h} & \text { in }(0, T) \times \Omega \\
\operatorname{div} \mathbf{u}=0 & \text { in }(0, T) \times \Omega \\
\mathbf{u}(x, t)=0 & \text { on }(0, T) \times \partial \Omega \\
\mathbf{u}(x, 0)=\mathbf{u}_{0}(x) & \text { in } \Omega
\end{array}
$$

\footnotetext{
2000 Mathematics Subject Classification. 35Q30,76D03, 76M99, 65M15.

Key words and phrases. Boussinesq equations, strong solutions, iterative method.

J.L. Boldrini was supported for this work by CAPES/MECD-DGU Brazil/Spain, Grant \#213705-4.

B. Climent-Ezquerra was supported for this work by CAPES/MECD-DGU Brazil/Spain, Grant \#2137-05-4.

M.D. Rojas-Medar is partially supported by Universidad de Antofagasta, Project PEI: 1333 and Fondecyt-Chile, Grant 1080628.

M. Rojas-Medar is partially supported by CAPES/MECD-DGU Brazil/Spain, grant \#213705-4, project BFM2003-06446-CO-01, Spain and Fondecyt-Chile, Grant 1080628.
} 


$$
\begin{array}{lr}
\frac{\partial \varphi}{\partial t}+\mathbf{u} \cdot \nabla \varphi-\operatorname{div}(k(\varphi) \nabla \varphi)=f & \text { in }(0, T) \times \Omega \\
\varphi(x, t)=\eta & \text { on }(0, T) \times \partial \Omega \\
\varphi(x, 0)=\varphi_{0}(x) & \text { in } \Omega
\end{array}
$$

Here $\mathbf{u}$ and $\varphi$ are associated respectively to the velocity and temperature of the fluid; $\nu(\varphi)$ and $k(\varphi)$ are associated respectively to the temperature dependent viscosity and heat conductivity; $\alpha>0$ is a constant associated to the coefficient of volume expansion; $\mathbf{g}, \mathbf{h}$ and $f$ are associated to given external forces. $\mathbf{u}_{0}(x)$ and $\varphi_{0}(x)$ are given initial data, and $\eta$ is a given boundary data for the temperature. The first equation comes from balance of linear momentum whereas the second one comes from the balance of internal energy.

We remark that these previous equations have much stronger nonlinearities than the classical Boussinesq approximations, and thus are much more difficult to handle.

Before we describe the contributions of the present article, let us briefly comment on previous related papers on this subject. When $\nu(\varphi)$ and $k(\varphi)$ are a positive constants, problem (1.1)-(1.2) reduces to the well studied classical Boussinesq model; for several results on this special situation, see for instance Morimoto [23], Hishida [12], Kagei [13]. Kagei and von Wahl [14], Málek, Ruzicka and Thäter [20]. For a closely related system modeling certain chemically active fluids, Rojas-Medar and Lorca [26], [27], [28], Moretti et al. [22], obtained results on existence of strong solutions.

The stationary problem associated to (1.1)-(1.2) was studied in [17] for bounded domains and in [24] for exterior domains. For the evolution model with temperature dependent viscosity and heat conductivity, (1.1)-(1.2), A.C. Moretti, M.A. RojasMedar and M.D. Rojas-Medar [21] proved existence of reproductive weak solutions in exterior domains; the existence of regular reproductive solution with Neumann condition on the temperature was studied by Climent-Ezquerra, Guillén-González and Rojas-Medar [5]; the case of periodic boundary conditions was studied by Feireisl and Málek in [7]. The existence of weak and strong solutions of the initial boundary value problem (1.1)-(1.2), was proved by Lorca and Boldrini in [18]. One interesting work is the one by Diaz and Galiano [3] where the more difficult case of fast or slow diffusion is discussed.

Our objective in the present work is to analyze an iterative scheme that can be used to find a sequence of approximate solutions that converge to a strong solution of (1.1)-(1.2). In fact, we will be able to estimate the rates of convergence of such approximate solutions in several norms.

The proposed iterative scheme is natural in the sense that it does something similar to what people interested in computational results would usually prefer: at each step one would like to solve a linear problem, and for this the information obtained in the previous step is used to linearize the problem. In our case, we compute the viscosity and the heat conductivity using the temperature obtained at the previous step, and thus at each step of our iterative method one has to solve in sequence two sets of linear partial differential equations: the first is a linear transport-diffusion equation, and the second is related to the Stokes problem. Our approximate solutions are the solutions of such linear problems.

Thus, although not yet at the complete discretization level, our results can be seen as a step in the direction of justifying this idea that the linearization done by using previously obtained information is suitable to obtain approximate solutions 
in the case of problem (1.1)-(1.2). We also hope that the techniques developed here could be adapted to the important case in which a full discretization is used; in this case, our results could also shed some light in what to expect in terms of the approximations rates.

We should remark that since this kind of iteration scheme is natural, it can be applied to other kind of systems. In fact, it was previously applied to a different fluid model (incompressible fluids with mass diffusion) in [9]. To prove the convergence of the sequence of approximate solutions, we use the same idea used in [9], that is, we prove that it is a Cauchy sequence in a suitable functional space. We also should stress, however, that since the our fluid model is different from the one in [9], and one of the nonlinearities present in (1.1)-(1.2) is really hard since it is in the higher order operator, the estimates necessary to complete the argument are very hard to obtain.

As a byproduct of our analysis we also obtain a result on existence and uniqueness of strong solutions of (1.1)-(1.2), which is similar to the one proved by Lorca and Boldrini in [18] by using a method different from the one used in the present article. Thus, a comparison between ours and the results of [18] is due.

We start such comparison by remarking that the theorem presented in [18] gives the local in time existence of strong solutions; that is, it requires that the time interval of existence be small; on the other hand, the result presented in the present work gives the of existence of solutions on a given interval of time $[0, T]$ by requiring smallness of the data. Since the result in [18] could be also stated in terms of existence of solutions on $[0, T]$, again by requiring smallness of the data, for the purposes of the comparisons that follow of assume it stated in this last form.

In [18], a sequence of approximate solutions constructed by the spectral FaedoGalekin method is used; that is, the eigenfunctions for the Laplace and the Stokes operators are used respectively as basis of the approximate temperature and fluid velocity. To obtain such approximate solutions, a coupled system of nonlinear differential equations had be analyzed; and suitable estimates had to be derived.

In the present work, as we previously said, we construct a sequence of approximate solutions by an iteration scheme, solving linear problems at each step, instead of nonlinear problems as in [18]. Since our main interest here is in deriving error estimates, which are not present in [18], we had to impose slightly more demanding conditions on the data than the ones required in [18]; for instance, we required that $\mathbf{g} \in L^{\infty}\left(0, T ; L^{6}(\Omega)\right), \mathbf{g}_{t} \in L^{4}\left(0, T ; L^{3}(\Omega)\right)$ and $\mathbf{u}_{0} \in V \cap H^{2}$ instead of $\mathbf{g} \in L^{\infty}\left(0, T ; L^{2}(\Omega)\right)$ and $\mathbf{u}_{0} \in V$ as in [18]. On the other hand, as expected, we obtain stronger solutions (see the definitions of these spaces in the next section.) For instance, we obtain that $\mathbf{u} \in L^{\infty}(0, T ; V \cap H)$ instead of just $\mathbf{u} \in L^{2}(0, T ; V \cap H)$ as in [18]. We also remark that in the usual applications, the gravitational field $\mathbf{g}$ is assumed constant, and thus the present hypotheses on $\mathbf{g}$ are not restrictive. Obviously, the same sort of differences appear for the respective approximate solutions.

We thus think that the present results can be seen as a step in the justification of the idea that the linearization using previously obtained information is a suitable way to obtain approximate solutions in the case of problem (1.1)-(1.2).

We also remark that, for simplicity, in the following we will consider homogeneous boundary conditions, that is, $\eta=0$; as usual, the non homogeneous case can be easily reduced to homogeneous one by assuming suitable smoothness on the 
boundary data and performing a trivial change of variable. By doing this we could have the statement on the existence of solutions as in [18],

Finally, we observe that the conditions we require for the viscosity and heat diffusion coefficient although nonlinear, do not allow both fast and slow diffusion. Thus, our results do not apply in the conditions of Diaz and Galiano [3], for instance, where estimates in strong norms are much more difficult to obtain.

The present work is organized as follows: in Section 2, we fix the notations, state the main hypotheses that hold throughout this work and describe the iterative approximation method to be used; we also state in this section our principal results concerning the existence and uniqueness of approximate and strong solutions, as well as our results on the associated rates of convergence. In Section 3, we give certain technical results that will be useful in the rest of the paper. Section 4 is dedicated to the proof of the existence of approximate solutions, while in Section 5 we prove the existence of solutions of the original problem and the convergence-rate bounds for the approximate solutions.

Finally, as it usual in this context, to ease the notation in computations of the estimates, $C, C_{1}, \ldots$ will denote generic positive constants depending only on the fixed data of the problem.

\section{Preliminaries, Hypotheses and Main Results}

We begin by fixing the notations and recalling certain definitions and facts to be used later in this paper. The $L^{2}(\Omega)$-product and norm are respectively denoted by $(\cdot, \cdot)$ and || ; the $L^{p}(\Omega)$-norm by ||$_{p}, 1 \leq p \leq \infty$; the $H^{m}(\Omega)$ - norm are denoted by \|\|$_{m}$ and the $W^{k, p}(\Omega)$-norm by ||$_{W^{k, p}}$. Here, $W^{k, p}(\Omega)$ is the usual Sobolev space; as usual, we denote $H^{m}(\Omega)=W^{m, 2}(\Omega)$, and $H_{0}^{1}(\Omega)$ is the closure of $C_{0}^{\infty}(\Omega)$ in the $H^{1}$ - norm. In general, the notation will be abridged: we set $L^{p}=L^{p}(\Omega), H_{0}^{1}=H_{0}^{1}(\Omega)$, and so on. Being $B$ a Banach space, we denote by $L^{q}(B)=L^{q}(0, T ; B)$ the Banach space of the B-valued functions defined in the interval $(0, T)$ that are $L^{q}$-integrable in the sense of Bochner. The functional spaces in this paper are either $\mathbb{R}$ or $\mathbb{R}^{3}$ - valued and will not distinguish these two situations in our notation. To which case we refer to will be clear from the context.

Let $C_{0, \sigma}^{\infty}(\Omega)=\left\{\mathbf{v} \in C_{0}^{\infty}(\Omega) ; \operatorname{div} \mathbf{v}=0\right\}, V=$ closure of $C_{0, \sigma}^{\infty}(\Omega)$ in $H_{0}^{1}(\Omega)$ and $H=$ closure of $C_{0, \sigma}^{\infty}(\Omega)$ in $L^{2}(\Omega)$. Let $P$ be the orthogonal projection from $L^{2}(\Omega)$ onto $H$ obtained by the usual Helmholtz decomposition. Then, the operator $A: H \rightarrow H$ given by $A=-P \Delta$ with domain $D(A)=H^{2}(\Omega) \cap V$ is called the Stokes operator. In order to obtain regularity properties of the Stokes operator we will assume that $\Omega$ is of class $C^{1,1}$ [1]. This assumption implies, in particular, that when $A \mathbf{u} \in L^{2}(\Omega)$, then $\mathbf{u} \in H^{2}(\Omega)$ and $\|\mathbf{u}\|_{H^{2}}$ and $|A \mathbf{u}|$ are equivalent norms.

We denote by $\left\{w^{k}\right\}_{k=1}^{\infty}$ the set of the eigenfunctions of the Stokes operator $A$, which are fields in $\left(H^{2}(\Omega)\right)^{n}$ that are complete orthogonal sets both in $H$ and $V$. We also denote $\left\{\psi^{k}\right\}_{k=1}^{\infty}$ the set of the eigenfunctions of the Laplace operator $-\Delta$ defined in $H_{0}^{1}(\Omega) \cap H^{2}(\Omega)$, which are functions in $H^{2}(\Omega) \cap H_{0}^{1}(\Omega)$ that are complete orthogonal sets both in $\left.L^{(} \Omega\right)$ and $H_{0}^{1}(\Omega)$. For each $j \in \mathbb{N}$, we define the finite dimensional subspaces $V_{j}=\operatorname{span}\left[w^{1}, \ldots, w^{j}\right] \subset V \cap\left(H^{2}(\Omega)\right)^{n}$ and $W_{j}=$ $\operatorname{span}\left[\psi^{1}, \ldots, \psi^{j}\right] \subset H_{0}^{1}(\Omega) \cap H^{2}(\Omega)$; we also considere the orthogonal projections $P_{j}: H \rightarrow V_{j}$ and $Q_{j} \rightarrow W_{j}$. 
Throughout the paper, we will suppose that $\nu, \nu^{\prime}, \nu^{\prime \prime}, k, k^{\prime}, k^{\prime \prime}$ are continuous functions such that for all $\varphi \in \mathbb{R}$

$$
\begin{array}{rlrl}
0<\nu_{0} \leq \nu(\varphi) & \leq \nu_{1}<+\infty, & 0<k_{0} \leq k(\varphi) \leq k_{1}<+\infty \\
\left|\nu^{\prime}(\varphi)\right| & \leq \nu_{1}^{\prime}<+\infty, \quad c \quad\left|k^{\prime}(\varphi)\right| \leq k_{1}^{\prime}<+\infty . \\
\left|\nu^{\prime \prime}(\varphi)\right| & \leq \nu_{1}^{\prime \prime}<+\infty, \quad & \left|k^{\prime \prime}(\varphi)\right| \leq k_{1}^{\prime \prime}<+\infty
\end{array}
$$

We stress that, even with the assumptions that the viscosity and the heat conduction coefficients are bounded functions of the temperature, the necessary mathematical analysis to obtain strong solutions for the present problem is very hard to do. In fact, excepting the easy lower order estimates required for weak solutions, the next usual higher order estimates, which are rather easy to derive in the case of constant viscosity and heat conduction, cannot be directly derived since the appearing extra terms are difficult to handle, as the reader will see in the coming computations.

We will use the following iterative process of the approximate solution of problem (1.1)-(1.2):

Initialization: Let $\mathbf{u}^{0}(t)=\mathbf{u}_{\mathbf{0}}$ and $\varphi^{0}(t)=\varphi_{\mathbf{0}}$ for all $t \in[0, T]$.

Step $n \geq 1$ : Firstly, given $\mathbf{u}^{n-1}$ and $\varphi^{n-1}$, we find $\varphi^{n}$ such that

$$
\begin{aligned}
& \varphi_{t}^{n}-\operatorname{div}\left(k\left(\varphi^{n-1}\right) \nabla \varphi^{n}\right)+\mathbf{u}^{n-1} \cdot \nabla \varphi^{n}=f, \\
& \varphi^{n}(x, 0)=\varphi_{0}(x) \text { in } \Omega, \\
& \varphi^{n}(x, t)=0 \text { on } \partial \Omega .
\end{aligned}
$$

Subsequently, known $\mathbf{u}^{n-1}, \varphi^{n-1}$ and $\varphi^{n}$, we find $\mathbf{u}^{n}, p^{n}$ such that

$$
\begin{aligned}
& \mathbf{u}_{t}^{n}-\operatorname{div}\left(\nu\left(\varphi^{n-1}\right) \nabla \mathbf{u}^{n}\right)+\mathbf{u}^{n-1} \cdot \nabla \mathbf{u}^{n}+\nabla p^{n}=\mathbf{h}+\alpha \varphi^{n} \mathbf{g}, \\
& \operatorname{div} \mathbf{u}^{n}=0, \\
& \mathbf{u}^{n}(x, 0)=\mathbf{u}_{0}(x) \quad \text { in } \Omega, \\
& \mathbf{u}^{n}(x, t)=0 \text { on } \partial \Omega .
\end{aligned}
$$

Recall that, for simplicity of exposition, we have taken homogeneous boundary conditions for the temperature. With this iterative scheme, we have reduced the nonlinear coupled system (1.1)-(1.2) to a sequence of linear problems.

Next, we state the main results of this paper. The first one is a result on existence and properties of the solutions of the previous approximate problems.

Theorem 2.1. Let $\Omega$ be a bounded domain in $\mathbb{R}^{N}(N=2$ or 3$)$ with a $C^{1,1}$ boundary and $0<T<+\infty$. Assume the following: $\nu$ and $k$ satisfy (2.1); $\mathrm{g} \in$ $L^{\infty}\left(L^{6}\right) ; f, \mathbf{h} \in L^{\infty}\left(L^{2}\right) ; \mathbf{g}_{t} \in L^{4}\left(L^{3}\right), f_{t}$ and $\mathbf{h}_{t} \in L^{2}\left(L^{2}\right)$. Then, if $\|f\|_{L^{\infty}\left(L^{2}\right)}$, $\|\mathbf{h}\|_{L^{\infty}\left(L^{2}\right)},\left\|f_{t}\right\|_{L^{2}\left(L^{2}\right)},\left\|\mathbf{h}_{\mathbf{t}}\right\|_{L^{2}\left(L^{2}\right)},\left\|\mathbf{u}_{0}\right\|_{H^{2} \cap V},\left\|\varphi_{0}\right\|_{H^{2} \cap H_{0}^{1}}$ and $\alpha$ are sufficiently small, the iteration scheme (2.2)-(2.3) generates a sequence $\left\{\left(\mathbf{u}^{n}, \varphi^{n}\right)\right\}_{n=0}^{\infty}$ such that $\mathbf{u}^{n} \in L^{\infty}(D(A)), \varphi^{n} \in L^{\infty}\left(H^{2}\right)$, and $\mathbf{u}_{t}^{n} \in L^{\infty}(H) \cap L^{2}(V), \varphi_{t}^{n} \in L^{\infty}\left(L^{2}\right) \cap L^{2}\left(H_{0}^{1}\right)$. Moreover, there is a positive constant $M$, which is independent of $n$, such that

$$
\begin{array}{cc}
\sup _{0 \leq t \leq T}\left|\nabla \varphi^{n}\right|^{2}<M & \sup _{0 \leq t \leq T}\left|\nabla \mathbf{u}^{n}\right|^{2}<M \\
\sup _{0 \leq t \leq T}\left|\Delta \varphi^{n}\right|^{2}<M & \sup _{0 \leq t \leq T}\left|A \mathbf{u}^{n}\right|^{2}<M \\
\sup _{0 \leq t \leq T}\left|\varphi_{t}^{n}\right|^{2}<M & \sup _{0 \leq t \leq T}\left|\mathbf{u}_{t}^{n}\right|^{2}<M \\
\int_{0}^{T}\left|\nabla \varphi_{t}^{n}\right|^{2}<M & \int_{0}^{T}\left|\nabla \mathbf{u}_{t}^{n}\right|^{2}<M,
\end{array}
$$


The next result is concerned with the existence of solutions of the original problem and the convergence-rates of the the approximate solutions.

Theorem 2.2. Under the conditions of Theorem 2.1, the approximate solutions $\left(\mathbf{u}^{n}, \varphi^{n}\right)$ converge in the space $L^{2}(D(A)) \times L^{2}\left(H^{2}\right)$ to $(\mathbf{u}, \varphi)$, which is a solution of problem

$$
\begin{aligned}
& \mathbf{u}_{t}-P(\operatorname{div}(\nu(\varphi) \nabla \mathbf{u}))+P(\mathbf{u} \cdot \nabla \mathbf{u})=\alpha P(\mathbf{g} \varphi)+P(\mathbf{h}), \\
& \varphi_{t}-\operatorname{div}(k(\varphi) \nabla \varphi)+\mathbf{u} \cdot \nabla \varphi=f,
\end{aligned}
$$

which, together with the associate pressure, is a strong solution of (1.1)-(1.2). This solution is unique and the same estimates stated in Theorem 2.1 hold for it; moreover, for all $n=1,2, \ldots$, we have the following rates of convergences for the approximate solutions:

$$
\begin{gathered}
\sup _{0 \leq \tau \leq t}\left\{\left|\mathbf{u}^{n}(\tau)-\mathbf{u}(\tau)\right|^{2}+\left|\varphi^{n}(\tau)-\varphi(\tau)\right|^{2}\right\} \leq C \frac{(D t)^{n}}{n !} \\
\sup _{0 \leq \tau \leq t}\left\{\int_{0}^{\tau}\left|\nabla \mathbf{u}^{n}(s)-\nabla \mathbf{u}(s)\right|^{2} d s+\int_{0}^{\tau}\left|\nabla \varphi^{n}(s)-\nabla \varphi(s)\right|^{2} d s\right\} \leq C \frac{(D t)^{n}}{n !}, \\
\sup _{0 \leq \tau \leq t}\left\{\left|\nabla \mathbf{u}^{n}(\tau)-\nabla \mathbf{u}(\tau)\right|^{2}+\left|\nabla \varphi^{n}(\tau)-\nabla \varphi(\tau)\right|^{2}\right\} \\
\leq C\left(\frac{(D t)^{n}}{n !}+\frac{(D t)^{n-1}}{(n-1) !}+\frac{(D t)^{(n-1) / 2}}{((n-1) / 2) !}\right) \\
\sup _{0 \leq \tau \leq t}\left\{\int_{0}^{\tau}\left|A \mathbf{u}^{n}(s)-A \mathbf{u}(s)\right|^{2} d s+\int_{0}^{\tau}\left|\Delta \varphi^{n}(s)-\Delta \varphi(s)\right|^{2} d s\right\} \\
\leq C\left(\frac{(D t)^{n}}{n !}+\frac{(D t)^{n-1}}{(n-1) !}+\frac{(D t)^{(n-1) / 2}}{((n-1) / 2) !}\right) \\
\sup _{0 \leq \tau \leq t}\left\{\int_{0}^{\tau}\left|\mathbf{u}_{t}^{n}(s)-\mathbf{u}_{t}(s)\right|^{2} d s+\int_{0}^{\tau}\left|\varphi_{t}^{n}(s)-\varphi t(s)\right|^{2} d s\right\} \\
\leq C\left(\frac{(D t)^{n}}{n !}+\frac{(D t)^{n-1}}{(n-1) !}+\frac{(D t)^{(n-1) / 2}}{((n-1) / 2) !}\right)
\end{gathered}
$$

where $C$ and $D$ are positive constants independent of $n$.

\section{Some TEChNicAl RESUlts}

We will need the following classical interpolation and Sobolev inequalities (for $3 D$ domains):

$$
|v|_{6} \leq C\|v\|_{1}, \quad|v|_{3} \leq C|v|^{1 / 2}\|v\|_{1}^{1 / 2}, \quad|v|_{\infty} \leq C\|v\|_{1}^{1 / 2}\|v\|_{2}^{1 / 2} .
$$

We will also need the results on the Helmholtz decomposition of $L^{2}$-vector fields stated in the following lemma. The first part of the lemma is well and its proof can be found for instance in Galdi [8] or Sohr [29]; the second part of the lemma is an estimate of the 'pressure' associated to the decomposition of a vector field $\mathbf{v}$ in terms of the $L^{2}$-norm of the Stokes operator applied to the field, but accompanied by a arbitrary small parameter. This last result, whose proof can be found in [17], will be important for the derivation of the required estimates of this paper. 
Lemma 3.1. Let $\mathbf{v} \in V \cap H^{2}$ and consider the Helmholtz decomposition of $-\Delta \mathbf{v}$, that is,

$$
-\Delta \mathbf{v}=A \mathbf{v}+\nabla q
$$

where $q \in H^{1}$ is taken such that $\int_{\Omega} q d x=0$. Then, there exists a positive constant $c$, depending only on $\Omega$. such that for all $\mathbf{v} \in V \cap H^{2}$ there holds

$$
\|q\|_{H^{1}} \leq c|A \mathbf{v}|
$$

Also, for each $\varepsilon>0$ there exists a positive constant $C_{\varepsilon}$, depending only on $\varepsilon$ and $\Omega$, such that for all $\mathbf{v} \in V \cap H^{2}$ we have

$$
|q| \leq C_{\varepsilon}|\nabla \mathbf{v}|+\varepsilon|A \mathbf{v}|
$$

Next, we present two estimates of Gronwall's type, which it will be used later on to obtain the required convergence rates.

The first result is similar to one firstly presented in [9], with the difference that for the result stated here the regularity conditions on the coefficients of the starting inequality are slightly stronger. As consequence, we are able to improve the exponent of the resulting inequality, which is important for our final results. The proof is similar to the one in [9], with suitable modifications; we do it here just for completeness.

Lemma 3.2. Let $a_{n}, b_{n}$ be two sequences of positive $L^{1}(0, T)$ functions such that for all $n \in \mathbb{N}$ there hold that $a_{n}(0) \leq A_{0} \in \mathbb{R}$, with $A_{0}$ independent of $n$, and

$$
a_{n}^{\prime}(t)+b_{n}(t) \leq c_{n}(t) a_{n}(t)+d_{n}(t) a_{n-1}(t), \quad \text { a.e. } t \in(0, T),
$$

where $c_{n}, d_{n}$ are two sequences of positive uniformly bounded functions in $L^{1}(0, T)$ and $L^{\infty}(0, T)$, respectively. Then, for $D=\max \left\{1,\left|d_{n}\right|_{\infty}\right\} e^{\max \left|c_{n}\right|_{1}}$, for all $n \in \mathbb{N}$ there holds:

$$
a_{n}(t)+\int_{0}^{t} b_{n}(s) d s \leq D A_{0} e^{D t}+\left|a_{0}\right|_{\infty} \frac{(D t)^{n}}{n !}, \quad \text { a.e.t } \in[0, T] .
$$

Proof. By applying the standard Gronwall's lemma to (3.2), we obtain

$$
a_{n}(t)+\int_{0}^{t} b_{n}(s) d s \leq\left(a_{n}(0)+\int_{0}^{t} d_{n}(s) a_{n-1}(s) d s\right) \exp \left(\int_{0}^{t} c_{n}(s) d s\right) .
$$

Thus, $a_{n}(t) \leq D\left(A_{0}+\int_{0}^{t} a_{n-1}(s) d s\right)$, and, by means an induction argument applying Fubini's theorem, we conclude that

$$
\begin{aligned}
a_{n}(t) & \leq D A_{0}\left(1+D t+\ldots .+\frac{(D t)^{n-1}}{(n-1) !}\right)+D^{n} \int_{0}^{t} \frac{(t-s)^{n-1}}{(n-1) !} a_{0}(s) d s \\
& \leq D A_{0} e^{D t}+\left|a_{0}\right|_{\infty} \frac{(D t)^{n}}{n !} .
\end{aligned}
$$

Next, by returning to (3.3) and applying our previous estimate for $a_{n-1}$, we obtain

$$
\int_{0}^{t} b_{n}(s) d s \leq D\left(A_{0}+\int_{0}^{t} a_{n-1}(s) d s\right) \leq D A_{0} e^{D t}+\left|a_{0}\right|_{\infty} \frac{(D t)^{n}}{n !},
$$

which concludes the proof. 
The next lemma is new and can be seen as a perturbation of the the previous one. It will be essential to the proof of the convergence of the approximate solutions.

Lemma 3.3. Let $a_{n}, b_{n}$ be two sequences of positive $L^{1}(0, T)$-functions such that for all $n \in \mathbb{N}$ there hold that $a_{n}(0)=0$ and

$$
a_{n}^{\prime}(t)+b_{n}(t) \leq c_{n}(t) a_{n}(t)+d_{n}(t) a_{n-1}(t)+\epsilon b_{n-1}(t), \quad \text { a.e. } t \in(0, T),
$$

where $c_{n}, d_{n}$ are two other sequences of positive uniformly bounded functions in $L^{\infty}(0, T)$ and $0 \leq \epsilon \leq 1 / 2$. Then, the series $\sum_{l=1}^{\infty}\left\|a_{l}\right\|_{L^{\infty}(0, T)}$ and $\sum_{l=1}^{\infty}\left\|b_{l}\right\|_{L^{1}(0, T)}$ are convergent. In particular, as $n \rightarrow+\infty$, we have $\sum_{l=n}^{\infty}\left\|a_{l}\right\|_{L^{\infty}(0, T)} \rightarrow 0$ and $\sum_{l=n}^{\infty}\left\|b_{l}\right\|_{L^{1}(0, T)} \rightarrow 0$

Proof. By integrating (3.4) from 0 to $t$ and adding the resulting inequalities from $l=1$ to $l=n$, we obtain

$$
\sum_{l=1}^{n} a_{l}(t)+\int_{0}^{t} \sum_{l=1}^{n} b_{l} \leq \int_{0}^{t} \sum_{l=1}^{n} c_{l} a_{l}+\int_{0}^{t} \sum_{l=1}^{n} d_{l} a_{l-1}+\epsilon \int_{0}^{t} \sum_{l=0}^{n-1} b_{l} .
$$

Since $0 \leq \epsilon \leq 1 / 2$, we obtain

$$
\sum_{l=1}^{n} a_{l}(t)+\frac{1}{2} \int_{0}^{t} \sum_{l=1}^{n} b_{l} \leq \int_{0}^{t} \sum_{l=1}^{n} c_{l} a_{l}+\int_{0}^{t} \sum_{l=0}^{n-1} d_{l+1} a_{l}+\frac{1}{2} \int_{0}^{t} b_{0}
$$

and because $c_{n}$ and $d_{n}$ uniformly bounded in $L^{\infty}(0, T)$, we get

$$
\sum_{l=1}^{n} a_{l}(t)+\frac{1}{2} \int_{0}^{t} \sum_{l=1}^{n} b_{l} \leq C \int_{0}^{t} \sum_{l=1}^{n} a_{l}+\frac{1}{2} \int_{0}^{t} b_{0} .
$$

Thus, by using Gronwall's lemma, we obtain that

$$
\sum_{l=1}^{n} a_{l}(t)+\frac{1}{2} \int_{0}^{t} \sum_{l=1}^{n} b_{l} \leq \frac{1}{2} e^{C t} \int_{0}^{t} b_{0}
$$

where the right hand side term is independent of $n$.

This implies that the series $\sum_{l=1}^{\infty}\left\|a_{l}\right\|_{L^{\infty}(0, T)}$ and $\sum_{l=1}^{\infty}\left\|b_{l}\right\|_{L^{1}(0, T)}$ are convergent, and we obtain in particular the stated results.

\section{Proof of Theorem 2.1}

We will prove Theorem 2.1 by using the spectral Galerkin method. In other words, fixed an integer $n \geq 1$, given $\mathbf{u}^{n-1} \in L^{\infty}(D(A))$ such that $\mathbf{u}_{t}^{n-1} \in L^{\infty}(H) \cap$ $L^{2}(V)$ and $\varphi^{n-1} \in L^{\infty}\left(H^{2}\right)$ such that $\varphi_{t}^{n-1} \in L^{\infty}\left(L^{2}\right) \cap L^{2}\left(H_{0}^{1}\right)$, for each $j \in \mathbb{N}$, we consider the following discrete variational formulations of (2.2) and (2.3).

Firstly find $\varphi_{j}^{n}(\cdot, t)=\Sigma_{i=1}^{j} c_{i}^{n, j}(t) \psi^{i}(\cdot) \in W_{j}$ such that $\forall v \in W_{j}$ :

$$
\begin{aligned}
& \left(\varphi_{j, t}^{n}, v\right)-\left(\operatorname{div}\left(k\left(\varphi^{n-1}\right) \nabla \varphi_{j}^{n}\right), v\right)+\left(\mathbf{u}^{n-1} \cdot \nabla \varphi^{n}, v\right)=(f, v), \\
& \left.\varphi^{n}\right|_{t=0}=Q_{j} \varphi_{0} .
\end{aligned}
$$


Subsequently, known $\mathbf{u}^{n-1}, \varphi^{n-1}$ and $\varphi^{n}$, we find $\mathbf{u}_{j}^{n}(\cdot, t)=\Sigma_{i=1}^{j} d_{i}^{n, j}(t) w^{i}(\cdot) \in$ $V_{j}$ such that for $\forall w \in V_{j}$

$$
\begin{aligned}
& \left(\mathbf{u}_{j, t}^{n}, w\right)-\left(\operatorname{div}\left(\nu\left(\varphi^{n-1}\right) \nabla \mathbf{u}_{j}^{n}\right), w\right)+\left(\mathbf{u}^{n-1} \cdot \nabla \mathbf{u}_{j}^{n}, w\right)=(\mathbf{h}, w)+\left(\alpha \varphi^{n} \mathbf{g}, w\right), \\
& \left.\mathbf{u}_{j}^{n}\right|_{t=0}=P_{j} \mathbf{u}_{0} .
\end{aligned}
$$

We remark that due to the regularity of the eigenvalues $w^{i}$ and $\psi^{i}, i=1, \ldots$, in these last two equations it is not required to pass the the divergence to the other term in the $L^{2}$-inner product.

We also observe that the last two equations actually correspond to two linear systems of ordinary differential equations for the coefficients $c_{i}^{n, j}$ and $d_{i}^{n, j}$, respectively. Under our conditions, the existence of solutions for such linear systems is the guaranteed on the time interval $[0, T]$. Besides, due to the known regularity of $\mathbf{u}^{n-1}, \varphi^{n-1}, k(\cdot), \nu(\cdot)$, the associated solutions $\mathbf{u}_{j}^{n}, \varphi_{j}^{n}$ have respectively at least the same regularity of $\mathbf{u}^{n-1}, \varphi^{n-1}$. Thus, the computations that we will have to perform in the next lemmas to obtain certain estimates for such solutions are justified.

For this, we will prove that the approximate solutions $\mathbf{u}^{n-1}, \varphi^{n-1}$ satisfy certain differential inequalities. In the sequel, $\delta$ and $\epsilon$ will denote small enough positive constants; $C$ will denote different positive constants depending only on the given data, on the chosen $\delta, \epsilon$ and the constants of the required interpolation inequalities.

We start with the following.

Lemma 4.1. Under the conditions of Theorem 2.1, $\mathbf{u}_{j}^{n}$ and $\varphi_{j}^{n}$ are uniformly bounded with respect to $j, n \in \mathbb{N}$ in $L^{\infty}(H) \cap L^{2}(V)$ and $L^{\infty}\left(L^{2}\right) \cap L^{2}\left(H_{0}^{1}\right)$, respectively.

Proof. By using $\varphi_{j}^{n}$ as a test function in (4.1), we obtain after some usual computations that

$$
\frac{d}{d t}\left|\varphi_{j}^{n}\right|^{2}+k_{0}\left|\nabla \varphi_{j}^{n}\right|^{2} \leq C|f|^{2}
$$

with a constant $C$ independent of $j$ and $n$.

By using $\mathbf{u}_{j}^{n}$ as a test function in (4.2), we find

$$
\frac{d}{d t}\left|\mathbf{u}_{j}^{n}\right|^{2}+\nu_{0}\left|\nabla \mathbf{u}_{j}^{n}\right|^{2} \leq C\left(|\mathbf{h}|^{2}+|\mathbf{g}|_{3}^{2}\left|\varphi_{j}^{n}\right|^{2}\right),
$$

again with a constant $C$ independent of $j$ and $n$. Thus, by adding (4.3) and (4.4) and applying the Gronwall's lemma, we get the stated result.

Lemma 4.2. Under the conditions of Theorem 2.1, there holds the following differential inequality with a positive constant $C$ independent of $j, n \in \mathbb{N}$ :

$$
\begin{aligned}
& \frac{d}{d t}\left(\left|\nabla \mathbf{u}_{j}^{n}\right|^{2}+\left|\nabla \varphi_{j}^{n}\right|^{2}\right)+\nu_{0}\left|A \mathbf{u}_{j}^{n}\right|^{2}+k_{0}\left|\Delta \varphi_{j}^{n}\right|^{2} \\
& \leq C\left(\left|\nabla \mathbf{u}^{n-1}\right|+\left|\Delta \varphi^{n-1}\right|\right)\left(\left|A \mathbf{u}_{j}^{n}\right|^{2}+\left|\Delta \varphi_{j}^{n}\right|^{2}\right) \\
& +C\left(|\mathbf{h}|^{2}+|f|^{2}+\alpha^{2}|\mathbf{g}|_{3}^{2}\left|\nabla \varphi_{j}^{n}\right|^{2}\right) .
\end{aligned}
$$

Proof. By taking $-\Delta \varphi_{j}^{n}$ as a test function in (4.1), which is possible because $-\Delta \varphi_{j}^{n} \in W_{j}$ due to the use of the spectral basis, and observing that div $\left(k\left(\varphi^{n-1}\right) \nabla \varphi_{j}^{n}\right)=$ $k^{\prime}\left(\varphi^{n-1}\right) \nabla \varphi^{n-1} \nabla \varphi_{j}^{n}+k\left(\varphi^{n-1}\right) \Delta \varphi_{j}^{n}$, we obtain 


$$
\begin{aligned}
\frac{1}{2} \frac{d}{d t}\left|\nabla \varphi_{j}^{n}\right|^{2}+k_{0}\left|\Delta \varphi_{j}^{n}\right|^{2} & \leq-\left(k^{\prime}\left(\varphi^{n-1}\right) \nabla \varphi^{n-1} \nabla \varphi_{j}^{n}, \Delta \varphi_{j}^{n}\right) \\
& +\left(\mathbf{u}^{n-1} \cdot \nabla \varphi_{j}^{n}, \Delta \varphi_{j}^{n}\right)-\left(f, \Delta \varphi_{j}^{n}\right) .
\end{aligned}
$$

The first term on the right hand side of (4.6) is bounded by

$$
\left|\left(k^{\prime}\left(\varphi^{n-1}\right) \nabla \varphi^{n-1} \nabla \varphi_{j}^{n}, \Delta \varphi_{j}^{n}\right)\right| \leq\left. k_{1}^{\prime}\left|\nabla \varphi^{n-1}{ }_{4}\right| \nabla \varphi_{j}^{n}\right|_{4}\left|\Delta \varphi_{j}^{n}\right| \leq C\left|\Delta \varphi^{n-1}\right|\left|\Delta \varphi_{j}^{n}\right|^{2} .
$$

Analogously, the second term in the right-hand side of (4.6) is estimated by

$$
\left|\left(\mathbf{u}^{n-1} \cdot \nabla \varphi_{j}^{n}, \Delta \varphi_{j}^{n}\right)\right| \leq C\left|\nabla u^{n-1}\right|\left|\Delta \varphi_{j}^{n}\right|^{2} .
$$

The last term in (4.6) is estimated by $C|f|^{2}+\left(k_{0} / 2\right)\left|\Delta \varphi_{j}^{n}\right|^{2}$.

Therefore, we obtain

$$
\frac{d}{d t}\left|\nabla \varphi_{j}^{n}\right|^{2}+k_{0}\left|\Delta \varphi_{j}^{n}\right|^{2} \leq C\left(\left|\Delta \varphi^{n-1}\right|+\left|\nabla \mathbf{u}^{n-1}\right|\right)\left|\Delta \varphi_{j}^{n}\right|^{2}+C|f|^{2} .
$$

Next, we take $A \mathbf{u}_{j}^{n}$ as a test function in (4.2) and use the Helmholtz decomposition of Lemma 3.1 to get

$$
\begin{aligned}
& \frac{1}{2} \frac{d}{d t}\left|\nabla \mathbf{u}_{j}^{n}\right|^{2}+\nu_{0}\left|A \mathbf{u}_{j}^{n}\right|^{2} \leq\left(\nu^{\prime}\left(\varphi^{n-1}\right) \nabla \varphi^{n-1} \nabla \mathbf{u}_{j}^{n}, A \mathbf{u}_{j}^{n}\right) \\
& \left.\stackrel{-}{-}\left(\varphi^{n-1}\right) \nabla q_{j}^{n}, A \mathbf{u}_{j}^{n}\right)-\left(\mathbf{u}^{n-1} \cdot \nabla \mathbf{u}_{j}^{n}, A \mathbf{u}_{j}^{n}\right)+\left(\mathbf{h}, A \mathbf{u}_{j}^{n}\right)+\alpha\left(\mathbf{g} \varphi_{j}^{n}, A \mathbf{u}_{j}^{n}\right) .
\end{aligned}
$$

In the same way as before, the first, third and fourth terms on the right-hand side of (4.8) can be bounded respectively by $C\left|\Delta \varphi^{n-1}\right|\left|A \mathbf{u}_{j}^{n}\right|^{2}, C\left|\nabla \mathbf{u}^{n-1}\right|\left|A \mathbf{u}_{j}^{n}\right|^{2}$ and $C|\mathbf{h}|^{2}+\left(\nu_{0} / 4\right)\left|A \mathbf{u}_{j}^{n}\right|^{2}$.

For the second term in the right-hand side of (4.8), we have

$$
\begin{aligned}
& -\left(\nu\left(\varphi^{n-1}\right) \nabla q_{j}^{n}, A \mathbf{u}_{j}^{n}\right)=-\left(q_{j}^{n}, \operatorname{div}\left(\nu\left(\varphi^{n-1}\right) A \mathbf{u}_{j}^{n}\right)\right) \\
& =-\left(q_{j}^{n}, \nu^{\prime}\left(\varphi^{n-1}\right) \nabla \varphi^{n-1} A \mathbf{u}_{j}^{n}\right)-\left(q_{j}^{n}, \nu\left(\varphi^{n-1}\right) \operatorname{div}\left(A \mathbf{u}_{j}^{n}\right)\right) \\
& \leq\left|-\left(q_{j}^{n}, \nu^{\prime}\left(\varphi^{n-1}\right) \nabla \varphi^{n-1} A \mathbf{u}_{j}^{n}\right)\right| \leq \nu_{1}^{\prime}\left|q_{j}^{n}\right|_{4}\left|\nabla \varphi^{n-1}\right|_{4}\left|A \mathbf{u}_{j}^{n}\right| \\
& \leq C\left|\nabla q_{j}^{n}\right|\left|\Delta \varphi^{n-1}\right|\left|A \mathbf{u}_{j}^{n}\right| \leq C\left|\Delta \varphi^{n-1}\right|\left|A \mathbf{u}_{j}^{n}\right|^{2} .
\end{aligned}
$$

The last term in (4.8) is estimated by

$$
\left|\alpha\left(\mathbf{g} \varphi_{j}^{n}, A \mathbf{u}_{j}^{n}\right)\right| \leq C \alpha^{2}\left|\nabla \varphi_{j}^{n}\right|^{2}|\mathbf{g}|_{3}^{2}+\frac{\nu_{0}}{4}\left|A \mathbf{u}_{j}^{n}\right|^{2} .
$$

Thus,

$$
\begin{aligned}
& \frac{d}{d t}\left|\nabla \mathbf{u}_{j}^{n}\right|^{2}+\nu_{0}\left|A \mathbf{u}_{j}^{n}\right|^{2} \leq C\left(\left|\nabla \mathbf{u}_{j}^{n-1}\right|+\left|\Delta \varphi^{n-1}\right|\right)\left|A \mathbf{u}_{j}^{n}\right|^{2} \\
& \quad+C\left(|\mathbf{h}|^{2}+\alpha^{2}|\mathbf{g}|_{3}^{2}\left|\nabla \varphi_{j}^{n}\right|^{2}\right),
\end{aligned}
$$

and by adding (4.7) and (4.9), we obtain the stated result.

Lemma 4.3. Under the conditions of Theorem 2.1, we have the following differential inequality with a positive constant $C$ independent of $j$ and $n \in \mathbb{N}$ :

$$
\begin{aligned}
& \frac{d}{d t}\left(\nu\left(\varphi^{n-1}\right)\left|\nabla \mathbf{u}_{j}^{n}\right|^{2}+k\left(\varphi^{n-1}\right)\left|\nabla \varphi_{j}^{n}\right|^{2}\right)+\left|\mathbf{u}_{j, t}^{n}\right|^{2}+\left|\varphi_{j, t}^{n}\right|^{2} \\
& \leq C\left(\left(\left|\nabla \mathbf{u}^{n-1}\right|+\left|\varphi_{t}^{n-1}\right|\right)\left(\left|A \mathbf{u}_{j}^{n}\right|^{2}+\left|\Delta \varphi_{j}^{n}\right|^{2}\right)\right. \\
& \left.+\left|\nabla \mathbf{u}^{n-1}\right|\left(\left|\varphi_{j, t}^{n}\right|^{2}+\left|\mathbf{u}_{j, t}^{n}\right|^{2}\right)\right)+C\left(|\mathbf{h}|^{2}+|f|^{2}+\alpha^{2}|\mathbf{g}|_{3}^{2}\left|\nabla \varphi_{j}^{n}\right|^{2}\right) .
\end{aligned}
$$


Proof. By taking $\varphi_{j, t}^{n} \in W_{j}$ as a test function in (4.1) and observing that

$$
\begin{aligned}
\left(k\left(\varphi^{n-1}\right) \nabla \varphi_{j}^{n}, \nabla \varphi_{j, t}^{n}\right)= & \frac{1}{2} \frac{d}{d t}\left(k\left(\varphi^{n-1}\right) \nabla \varphi_{j}^{n}, \nabla \varphi_{j}^{n}\right) \\
& -\frac{1}{2}\left(k^{\prime}\left(\varphi^{n-1}\right) \varphi_{t}^{n-1} \nabla \varphi_{j}^{n}, \nabla \varphi_{j}^{n}\right),
\end{aligned}
$$

we obtain

$$
\begin{aligned}
\frac{1}{2} \frac{d}{d t}\left(k\left(\varphi^{n-1}\right)\left|\nabla \varphi_{j}^{n}\right|^{2}\right)+\left|\varphi_{j, t}^{n}\right|^{2} & \leq \frac{1}{2}\left(k^{\prime}\left(\varphi^{n-1}\right) \varphi_{t}^{n-1} \nabla \varphi_{j}^{n}, \nabla \varphi_{j}^{n}\right) \\
& +\left(\mathbf{u}^{n-1} \cdot \nabla \varphi_{j}^{n}, \varphi_{j, t}^{n}\right)+\left(f, \varphi_{j, t}^{n}\right) .
\end{aligned}
$$

The first term on the right-hand side of (4.11) is bounded by

$$
\left|\left(k^{\prime}\left(\varphi^{n-1}\right) \varphi_{t}^{n-1} \nabla \varphi_{j}^{n}, \nabla \varphi_{j}^{n}\right)\right| \leq k_{1}^{\prime}\left|\varphi_{t}^{n-1}\right|_{2}\left|\nabla \varphi_{j}^{n}\right|_{4}^{2} \leq C\left|\varphi_{t}^{n-1}\right|\left|\Delta \varphi_{j}^{n}\right|^{2} .
$$

The second term in the right-hand side of (4.11) is estimated by

$$
\begin{gathered}
\left|\left(\mathbf{u}^{n-1} \cdot \nabla \varphi_{j}^{n}, \varphi_{j, t}^{n}\right)\right| \leq C\left|\mathbf{u}^{n-1}\right|_{4}\left|\nabla \varphi_{j}^{n}\right|_{4}\left|\varphi_{j, t}^{n}\right| \leq C\left|\nabla \mathbf{u}^{n-1}\right|\left|\Delta \varphi_{j}^{n}\right|\left|\varphi_{j, t}^{n}\right| \\
\leq C\left(\left|\nabla \mathbf{u}^{n-1}\right|\left|\Delta \varphi_{j}^{n}\right|^{2}+\left|\nabla \mathbf{u}^{n-1}\right|\left|\varphi_{j, t}^{n}\right|^{2}\right) .
\end{gathered}
$$

The last term of (4.11) is bounded by $C|f|^{2}+(1 / 2)\left|\varphi_{j, t}^{n}\right|^{2}$. Hence,

$$
\begin{aligned}
\frac{d}{d t}\left(k\left(\varphi^{n-1}\right)\left|\nabla \varphi_{j}^{n}\right|^{2}\right)+\left|\varphi_{j, t}^{n}\right|^{2} \leq & C\left(\left|\varphi_{j, t}^{n-1}\right|+\left|\nabla \mathbf{u}^{n-1}\right|\right)\left|\Delta \varphi_{j}^{n}\right|^{2} \\
& +C\left|\nabla \mathbf{u}^{n-1}\right|\left|\varphi_{j, t}^{n}\right|^{2}+\frac{1}{2}|f|^{2} .
\end{aligned}
$$

Next, we take $\mathbf{u}_{t}^{n} \in V_{j}$ as a test function in (4.2) and observe that

$$
\left(\nu\left(\varphi^{n-1}\right) \nabla \mathbf{u}_{j}^{n}, \nabla \mathbf{u}_{j, t}^{n}\right)=\frac{1}{2} \frac{d}{d t}\left(\nu\left(\varphi^{n-1}\right) \nabla \mathbf{u}_{j}^{n}, \nabla \mathbf{u}_{j}^{n}\right)-\frac{1}{2}\left(\nu^{\prime}\left(\varphi^{n-1}\right) \varphi_{t}^{n-1} \nabla \mathbf{u}_{j}^{n}, \nabla \mathbf{u}_{j}^{n}\right)
$$

to obtain

$$
\begin{array}{r}
\frac{1}{2} \frac{d}{d t}\left(\nu\left(\varphi^{n-1}\right)\left|\nabla \mathbf{u}_{j}^{n}\right|^{2}\right)+\left|\mathbf{u}_{j, t}^{n}\right|^{2} \leq \frac{1}{2}\left(\nu^{\prime}\left(\varphi^{n-1}\right) \varphi_{t}^{n-1} \nabla \mathbf{u}_{j}^{n}, \nabla \mathbf{u}_{j}^{n}\right) \\
-\left(\mathbf{u}^{n-1} \cdot \nabla \mathbf{u}_{j}^{n}, \mathbf{u}_{j, t}^{n}\right)+\left(\mathbf{h}, \mathbf{u}_{j, t}^{n}\right)+\alpha\left(\mathbf{g} \varphi_{j}^{n}, \mathbf{u}_{j, t}^{n}\right) .
\end{array}
$$

Next, we observe that the first three terms on the right-hand side of (4.13) can be estimated respectively by $C\left|\varphi_{t}^{n-1}\right|\left|A \mathbf{u}_{j}^{n}\right|^{2}, C\left(\left|\nabla \mathbf{u}^{n-1}\right|\left|A \mathbf{u}_{j}^{n}\right|^{2}+\left|\nabla \mathbf{u}^{n-1}\right|\left|\mathbf{u}_{j, t}^{n}\right|^{2}\right)$ and $C|\mathbf{h}|^{2}+\frac{1}{4}\left|\mathbf{u}_{j, t}^{n}\right|^{2}$. The last term is estimated as

$$
\left|\alpha\left(\mathbf{g} \varphi_{j}^{n}, \mathbf{u}_{j, t}^{n}\right)\right| \leq \alpha|\mathbf{g}|_{3}\left|\varphi_{j}^{n}\right|_{6}\left|\mathbf{u}_{j, t}^{n}\right| \leq C \alpha^{2}|\mathbf{g}|_{3}^{2}\left|\nabla \varphi_{j}^{n}\right|^{2}+\frac{1}{4}\left|\mathbf{u}_{j, t}^{n}\right|^{2} .
$$

Thus, (4.13) implies

$$
\begin{array}{r}
\frac{d}{d t}\left(\nu\left(\varphi^{n-1}\right)\left|\nabla \mathbf{u}_{j}^{n}\right|^{2}\right)+\left|\mathbf{u}_{j, t}^{n}\right|^{2} \leq C\left(\left|\nabla \mathbf{u}^{n-1}\right|+\left|\varphi_{t}^{n-1}\right|\right)\left|A \mathbf{u}_{j}^{n}\right|^{2} \\
+\left|\nabla \mathbf{u}^{n-1}\right|\left|\mathbf{u}_{j, t}^{n}\right|^{2}+C\left(|\mathbf{h}|^{2}+\alpha^{2}|\mathbf{g}|_{3}^{2}\left|\nabla \varphi_{j}^{n}\right|_{2}^{2}\right) .
\end{array}
$$

By adding (4.12) and (4.14), we then get the stated result.

Lemma 4.4. Under the conditions of Theorem 2.1, there holds the following differential inequality with a positive constant $C$ independent of $j$ and $n \in \mathbb{N}$ :

$$
\begin{array}{r}
\frac{d}{d t}\left(\left|\mathbf{u}_{j, t}^{n}\right|^{2}+\left|\varphi_{j, t}^{n}\right|^{2}\right)+\nu_{0}\left|\nabla \mathbf{u}_{j, t}^{n}\right|^{2}+k_{0}\left|\nabla \varphi_{j, t}^{n}\right|^{2} \leq \frac{1}{2}\left|\mathbf{u}_{j, t}^{n}\right|^{2}+\frac{1}{4}\left|\varphi_{j, t}^{n}\right|^{2} \\
+\frac{k_{0}}{2}\left|\nabla \varphi_{j, t}^{n}\right|^{2}+C\left(\left|\nabla \mathbf{u}_{t}^{n-1}\right|+\left|\nabla \varphi_{t}^{n-1}\right|\right)\left(\left|A \mathbf{u}_{j}^{n}\right|^{2}+\left|\Delta \varphi_{j}^{n}\right|^{2}\right) \\
\left.+C\left(\alpha^{4}|\mathbf{g}|_{6}^{4}\left|\varphi_{j, t}^{n}\right|^{2}+\alpha^{2}\left|\mathbf{g}_{t}\right|_{3}^{2}\left|\nabla \varphi_{j}^{n}\right|^{2}\right)\right)+2\left(\left|\mathbf{h}_{t}\right|^{2}+\left|f_{t}\right|^{2}\right) .
\end{array}
$$


Proof. By differentiating (4.1) with respect to the time and using $\varphi_{j, t}^{n} \in W_{j}$ as a test function, we obtain

$$
\begin{aligned}
\frac{1}{2} \frac{d}{d t}\left|\varphi_{j, t}^{n}\right|^{2}+k_{0}\left|\nabla \varphi_{j, t}^{n}\right|^{2} & \leq-\left(k^{\prime}\left(\varphi^{n-1}\right) \varphi_{t}^{n-1} \nabla \varphi_{j}^{n}, \nabla \varphi_{j, t}^{n}\right) \\
& -\left(\mathbf{u}_{t}^{n-1} \cdot \nabla \varphi_{j}^{n}, \varphi_{j, t}^{n}\right)+\left(f_{t}, \varphi_{j, t}^{n}\right) .
\end{aligned}
$$

The first term on the right hand side of (4.16) is bounded by

$$
\begin{gathered}
\left|-\left(k^{\prime}\left(\varphi^{n-1}\right) \varphi_{t}^{n-1} \nabla \varphi_{j}^{n}, \nabla \varphi_{j, t}^{n}\right)\right| \leq k_{1}^{\prime}\left|\varphi_{t}^{n-1}\right|_{4}\left|\nabla \varphi_{j}^{n}\right|_{4}\left|\nabla \varphi_{j, t}^{n}\right| \\
\leq \frac{k_{0}}{2}\left|\nabla \varphi_{j, t}^{n}\right|^{2}+C\left|\nabla \varphi_{t}^{n-1}\right|^{2}\left|\Delta \varphi_{j}^{n}\right|^{2} .
\end{gathered}
$$

Analogously, the second term is bounded by

$$
\left|-\left(\mathbf{u}_{t}^{n-1} \cdot \nabla \varphi_{j}^{n}, \varphi_{j, t}^{n}\right)\right| \leq \frac{1}{8}\left|\varphi_{j, t}^{n}\right|^{2}+C\left|\nabla \mathbf{u}_{t}^{n-1}\right|^{2}\left|\Delta \varphi_{j}^{n}\right|^{2} .
$$

The last term is bounded by $2\left|f_{t}\right|^{2}+\frac{1}{8}\left|\varphi_{j, t}^{n}\right|^{2}$.

From these previous estimates, we then get that

$$
\begin{aligned}
\frac{d}{d t}\left|\varphi_{j, t}^{n}\right|^{2}+k_{0}\left|\nabla \varphi_{j, t}^{n}\right|^{2} & \leq \frac{1}{4}\left|\varphi_{j, t}^{n}\right|^{2} \\
& +C\left(\left|\nabla \mathbf{u}_{t}^{n-1}\right|^{2}+\left|\nabla \varphi_{t}^{n-1}\right|^{2}\right)\left|\Delta \varphi_{j}^{n}\right|^{2}+2\left|f_{t}\right|^{2}
\end{aligned}
$$

By differentiating (4.2) with respect to the time and using $\mathbf{u}_{j, t}^{n} \in V_{j}$ as a test function, we get

$$
\begin{aligned}
& \frac{1}{2} \frac{d}{d t}\left(\left|\mathbf{u}_{j, t}^{n}\right|^{2}\right)+\nu_{0}\left|\nabla \mathbf{u}_{j, t}^{n}\right|^{2} \leq-\left(\nu^{\prime}\left(\varphi^{n-1}\right) \varphi_{t}^{n-1} \nabla \mathbf{u}_{j}^{n}, \nabla \mathbf{u}_{j, t}^{n}\right) \\
& -\left(\mathbf{u}_{t}^{n-1} \cdot \nabla \mathbf{u}_{j}^{n}, \mathbf{u}_{j, t}^{n}\right)+\left(\mathbf{h}_{t}, \mathbf{u}_{j, t}^{n}\right)+\alpha\left(\mathbf{g} \varphi_{j, t}^{n}, \mathbf{u}_{j, t}^{n}\right)+\alpha\left(\mathbf{g}_{t} \varphi^{n}, \mathbf{u}_{j, t}^{n}\right) .
\end{aligned}
$$

As in the previous inequality, the first three terms on the right hand side of (4.18) can be bounded respectively by $\frac{\nu_{0}}{2}\left|\nabla \mathbf{u}_{j, t}^{n}\right|_{2}^{2}+C\left|\nabla \varphi_{t}^{n-1}\right|\left|A \mathbf{u}_{j}^{n}\right|^{2}, \frac{1}{8}\left|\nabla \mathbf{u}_{j, t}^{n}\right|_{2}^{2}+$ $C\left|\nabla \mathbf{u}_{t}^{n-1}\right|\left|A \mathbf{u}_{j}^{n}\right|^{2}$ and $\frac{1}{8}\left|\mathbf{u}_{j, t}^{n}\right|^{2}+2\left|\mathbf{h}_{t}\right|^{2}$.

For the last two terms, we observe that:

$$
\begin{gathered}
\left|\alpha\left(\mathbf{g} \varphi_{j, t}^{n}, \mathbf{u}_{j, t}^{n}\right)\right| \leq \alpha|\mathbf{g}|_{6}\left|\varphi_{j, t}^{n}\right|_{3}\left|\mathbf{u}_{j, t}^{n}\right| \leq \frac{1}{8}\left|\mathbf{u}_{j, t}^{n}\right|^{2}+\frac{k_{0}}{2}\left|\nabla \varphi_{j, t}^{n}\right|^{2}+C \alpha^{4}|\mathbf{g}|_{6}^{4}\left|\varphi_{j, t}^{n}\right|^{2}, \\
\left|\alpha\left(\mathbf{g}_{t} \varphi_{j}^{n}, \mathbf{u}_{j, t}^{n}\right)\right| \leq \alpha\left|\varphi_{j}^{n}\right|_{6}\left|\mathbf{g}_{t}\right|_{3}\left|\mathbf{u}_{j, t}^{n}\right| \leq \frac{1}{8}\left|\mathbf{u}_{j, t}^{n}\right|^{2}+C \alpha^{2}\left|\mathbf{g}_{t}\right|_{3}^{2}\left|\nabla \varphi_{j}^{n}\right|^{2}
\end{gathered}
$$

$$
\begin{aligned}
\frac{d}{d t}\left|\mathbf{u}_{j, t}^{n}\right|^{2}+\nu_{0}\left|\nabla \mathbf{u}_{j, t}^{n}\right|^{2} & \leq \frac{1}{2}\left|\mathbf{u}_{j, t}^{n}\right|^{2}+\frac{k_{0}}{2}\left|\nabla \varphi_{j, t}^{n}\right|^{2}+C\left(\left|\nabla \mathbf{u}^{n-1}\right|+\left|\varphi_{t}^{n-1}\right|\right)\left|A \mathbf{u}_{j}^{n}\right|^{2} \\
& +C\left(\alpha^{4}|\mathbf{g}|_{6}^{4}\left|\varphi_{j, t}^{n}\right|^{2}+\alpha^{2}\left|\mathbf{g}_{t}\right|_{3}^{2}\left|\nabla \varphi_{j}^{n}\right|^{2}\right)+2\left|\mathbf{h}_{t}\right|^{2} .
\end{aligned}
$$

Finally, by adding (4.17) and (4.19), we get (4.15) .

Lemma 4.5. Under the conditions of Theorem 2.1, we have the following differential inequality with a positive constants $C_{\varphi}, C_{u}$ and $C$, which are independent of 
$j$ and $n \in \mathbb{N}:$

$$
\begin{aligned}
& \left(\frac{k_{0}}{2}-C_{\varphi}\left|\Delta \varphi^{n-1}\right|-C_{\varphi}\left|A \mathbf{u}^{n-1}\right|\right)\left|\Delta \varphi_{j}^{n}\right|^{2} \\
& +\left(\frac{\nu_{0}}{2}-C_{u}\left|\Delta \varphi^{n-1}\right|-C_{u}\left|A \mathbf{u}^{n-1}\right|\right)\left|A \mathbf{u}_{j}^{n}\right|^{2} \\
& \leq C\left(\left|\mathbf{u}_{j, t}^{n}\right|^{2}+\left|\varphi_{j, t}^{n}\right|^{2}+|\mathbf{g}|_{4}^{2}\left|\nabla \varphi_{j}^{n}\right|^{2}\right)+C\left(|\mathbf{h}|^{2}+|f|^{2}\right) .
\end{aligned}
$$

Proof. By taking $-\Delta \varphi_{j}^{n} \in W_{j}$ as a test function in (4.1), we obtain

$$
\begin{array}{r}
\left(k\left(\varphi^{n-1}\right) \Delta \varphi_{j}^{n}, \Delta \varphi_{j}^{n}\right)=\left(\varphi_{j, t}^{n}, \Delta \varphi_{j}^{n}\right)-\left(k^{\prime}\left(\varphi^{n-1}\right) \nabla \varphi^{n-1} \nabla \varphi_{j}^{n}, \Delta \varphi_{j}^{n}\right) \\
+\left(\mathbf{u}^{n-1} \cdot \nabla \varphi_{j}^{n}, \Delta \varphi_{j}^{n}\right)-\left(f, \Delta \varphi_{j}^{n}\right) .
\end{array}
$$

By estimating the right-hand side of (4.21), we get

$$
\left(\frac{k_{0}}{2}-C_{\varphi}\left|\Delta \varphi^{n-1}\right|-C_{\varphi}\left|A \mathbf{u}^{n-1}\right|\right)\left|\Delta \varphi_{j}^{n}\right|^{2} \leq C\left(\left|\varphi_{j, t}^{n}\right|^{2}+|f|^{2}\right) .
$$

Next, by taking $A u_{j}^{n} \in V_{j}$ as a test function in (4.2) and using the Helmholtz decomposition of lemma 3.1 , we obtain for some $q_{j}^{n}$

$$
\begin{array}{r}
\left(\nu\left(\varphi^{n-1}\right) A \mathbf{u}_{j}^{n}, A \mathbf{u}_{j}^{n}\right) \leq-\left(\mathbf{u}_{j, t}^{n}, A \mathbf{u}_{j}^{n}\right)+\left(\nu^{\prime}\left(\varphi^{n-1}\right) \nabla \varphi^{n-1} \nabla \mathbf{u}_{j}^{n}, A \mathbf{u}_{j}^{n}\right) \\
-\left(q_{j}^{n}, \nu\left(\varphi^{n-1}\right) \nabla \varphi^{n-1} A \mathbf{u}_{j}^{n}\right)-\left(\mathbf{u}^{n-1} \cdot \nabla \mathbf{u}_{j}^{n}, A \mathbf{u}_{j}^{n}\right) \\
+\left(\mathbf{h}, A \mathbf{u}_{j}^{n}\right)+\alpha\left(\mathbf{g} \varphi_{j}^{n}, A \mathbf{u}_{j}^{n}\right) .
\end{array}
$$

Similarly as above, by bounding the right-hand side of (4.23), using inequality (3.1) in Lemma 3.1 with a suitable small $\varepsilon>0$, we find:

$$
\begin{aligned}
& \left(\frac{\nu_{0}}{2}-C_{u}\left|\Delta \varphi^{n-1}\right|-C_{u}\left|A \mathbf{u}^{n-1}\right|\right)\left|A \mathbf{u}_{j}^{n}\right|^{2} \\
& \leq C\left(\left|\mathbf{u}_{j, t}^{n}\right|^{2}+|\mathbf{g}|_{4}^{2}\left|\nabla \varphi_{j}^{n}\right|^{2}+|\mathbf{h}|^{2}\right)
\end{aligned}
$$

By adding (4.21) and (4.24), we obtain (4.20).

In the next lemma we will combine the previous result to obtain uniform estimates under certain conditions.

Lemma 4.6. There is a constant $M$, independent of $j$ and $n \in \mathbb{N}$, such that if

$$
\begin{array}{ll}
\sup _{0 \leq t \leq T}\left|\nabla \varphi^{n-1}\right|^{2}<M, & \sup _{0 \leq t \leq T}\left|\nabla \mathbf{u}^{n-1}\right|^{2}<M, \\
\sup _{0 \leq t \leq T}\left|\Delta \varphi^{n-1}\right|^{2}<M, & \sup _{0 \leq t \leq T}\left|A \mathbf{u}^{n-1}\right|^{2}<M, \\
\sup _{0 \leq t \leq T}\left|\varphi_{t}^{n-1}\right|^{2}<M, & \sup _{0 \leq t \leq T}\left|\mathbf{u}_{t}^{n-1}\right|^{2}<M, \\
\int_{0}^{T}\left|\nabla \varphi_{t}^{n-1}\right|^{2}<M, & \int_{0}^{T}\left|\nabla \mathbf{u}_{t}^{n-1}\right|^{2}<M
\end{array}
$$

we also have

$$
\begin{array}{cc}
\sup _{0 \leq t \leq T}\left|\nabla \varphi_{j}^{n}\right|^{2}<M & \sup _{0 \leq t \leq T}\left|\nabla \mathbf{u}_{j}^{n}\right|^{2}<M \\
\sup _{0 \leq t \leq T}\left|\Delta \varphi_{j}^{n}\right|^{2}<M & \sup _{0 \leq t \leq T}\left|A \mathbf{u}_{j}^{n}\right|^{2}<M \\
\sup _{0 \leq t \leq T}\left|\varphi_{j, t}^{n}\right|^{2}<M & \sup _{0 \leq t \leq T}\left|\mathbf{u}_{j, t}^{n}\right|^{2}<M \\
\int_{0}^{T}\left|\nabla \varphi_{j, t}^{n}\right|^{2}<M & \int_{0}^{T}\left|\nabla \mathbf{u}_{j, t}^{n}\right|^{2}<M,
\end{array}
$$

for small enough $\|f\|_{L^{\infty}\left(L^{2}\right)},\|\mathbf{h}\|_{L^{\infty}\left(L^{2}\right)},\left\|f_{t}\right\|_{L^{2}\left(L^{2}\right)},\left\|\mathbf{h}_{\mathbf{t}}\right\|_{L^{2}\left(L^{2}\right)},\left\|\mathbf{u}_{0}\right\|_{H^{2} \cap V},\left\|\varphi_{0}\right\|_{H^{2} \cap H_{0}^{1}}$ and $\alpha$, again independently of $j$ and $n \in \mathbb{N}$. 
Proof. We start by adding (4.5), (4.10) and (4.15) to get

$$
\begin{gathered}
\frac{d}{d t}\left(\left(1+\nu\left(\varphi^{n-1}\right)\right)\left|\nabla \mathbf{u}_{j}^{n}\right|^{2}+\left(1+k\left(\varphi^{n-1}\right)\right)\left|\nabla \varphi_{j}^{n}\right|^{2}+\left|\mathbf{u}_{j, t}^{n}\right|^{2}+\left|\varphi_{j, t}^{n}\right|^{2}\right) \\
+C_{1}\left(\left|\Delta \varphi_{j}^{n}\right|^{2}+\left|A \mathbf{u}_{j}^{n}\right|^{2}+\left|\varphi_{j, t}^{n}\right|^{2}+\left|\mathbf{u}_{j, t}^{n}\right|^{2}+\left|\nabla \varphi_{j, t}^{n}\right|^{2}+\left|\nabla \mathbf{u}_{j, t}^{n}\right|^{2}\right) \\
\leq C_{2}\left(\left|\Delta \varphi^{n-1}\right|^{2}+\left|\nabla \mathbf{u}^{n-1}\right|^{2}+\left|\varphi_{t}^{n-1}\right|^{2}\right)\left(\left|\Delta \varphi_{j}^{n}\right|^{2}+\left|A \mathbf{u}_{j}^{n}\right|^{2}\right) \\
+C_{2}\left(\left|\nabla \mathbf{u}_{t}^{n-1}\right|^{2}+\left|\nabla \varphi_{t}^{n-1}\right|^{2}\right)\left(\left|\Delta \varphi_{j}^{n}\right|^{2}+\left|A \mathbf{u}_{j}^{n}\right|^{2}\right) \\
+C_{2}\left|\nabla \mathbf{u}^{n-1}\right|\left(\left|\mathbf{u}_{j, t}^{n}\right|^{2}+\left|\varphi_{j, t}^{n}\right|^{2}\right)+C_{2} \alpha^{4}|\mathbf{g}|_{6}^{4}\left|\varphi_{j, t}^{n}\right|^{2} \\
+C_{2} \alpha^{2}\left(|\mathbf{g}|_{3}^{2}+\left|\mathbf{g}_{t}\right|_{3}^{2}\right)\left|\nabla \varphi_{j}^{n}\right|^{2}+C_{2}\left(|f|^{2}+|\mathbf{h}|^{2}+\left|f_{t}\right|^{2}+\left|\mathbf{h}_{t}\right|^{2}\right),
\end{gathered}
$$

where the positive constants $C_{1}$ and $C_{2}$ are independent of $j$ and $n \in \mathbb{N}$.

By integrating in time, after some manipulations, the inequality (4.27) gives:

$$
\begin{aligned}
& \left|\nabla \mathbf{u}_{j}^{n}\right|^{2}+\left|\nabla \varphi_{j}^{n}\right|^{2}+\left|\mathbf{u}_{j, t}^{n}\right|^{2}+\left|\varphi_{j, t}^{n}\right|^{2} \\
& +\int_{0}^{t}\left[C_{1}-C_{2}\left(\left|\Delta \varphi^{n-1}\right|+\left|\nabla \mathbf{u}^{n-1}\right|+\left|\varphi_{t}^{n-1}\right|\right]\left(\left|\Delta \varphi_{j}^{n}\right|^{2}+\left|A \mathbf{u}_{j}^{n}\right|^{2}\right)\right) \\
& +C_{1} \int_{0}^{t}\left(\left|\varphi_{j, t}^{n}\right|^{2}+\left|\mathbf{u}_{j, t}^{n}\right|^{2}+\left|\nabla \varphi_{j, t}^{n}\right|^{2}+\left|\nabla \mathbf{u}_{j, t}^{n}\right|^{2}\right) \\
& \leq C_{3}\left(\left|\nabla \mathbf{u}_{j}^{n}(0)\right|^{2}+\left|\nabla \varphi_{j}^{n}(0)\right|^{2}+\left|\mathbf{u}_{j, t}^{n}(0)\right|^{2}+\left|\varphi_{j, t}^{n}(0)\right|^{2}\right) \\
& +C_{2}\left(\int_{0}^{t}\left|\nabla \mathbf{u}_{t}^{n-1}\right|^{2}+\left|\nabla \varphi_{t}^{n-1}\right|^{2}\right)\left(\left|\Delta \varphi_{j}^{n}\right|^{2}+\left|A \mathbf{u}_{j}^{n}\right|^{2}\right) \\
& +C_{2} \int_{0}^{t}\left(\left|\nabla \mathbf{u}^{n-1}\right|+\alpha^{4}|\mathbf{g}|_{6}^{4}+\alpha^{2}|\mathbf{g}|_{3}^{2}+\alpha^{2}\left|\mathbf{g}_{t}\right|_{3}^{2}\right)\left(\left|\nabla \mathbf{u}_{j}^{n}\right|^{2}+\left|\nabla \varphi_{j}^{n}\right|^{2}+\left|\mathbf{u}_{j, t}^{n}\right|^{2}+\left|\varphi_{j, t}^{n}\right|^{2}\right) \\
& +C_{2} \int^{2}\left(|f|^{2}+|\mathbf{h}|^{2}+\left|f_{t}\right|^{2}+\left|\mathbf{h}_{t}\right|^{2}\right),
\end{aligned}
$$

again with the positive constants $C_{1}, C_{2}$ and $C_{3}$ independent of $j$ and $n \in \mathbb{N}$.

Next, we fix $\delta$ such that

$$
0<\delta<\min \left\{1, \frac{k_{0}}{8 C_{\varphi}}, \frac{\nu_{0}}{8 C_{u}}, \frac{C_{1}}{12 C_{2}}, \frac{\ln 3-1}{(2+T) C_{5}}\right\}
$$

where the constants $C_{\varphi}$ and $C_{u}$ are those appearing in (4.20) and $C$ and $C_{1}$ are those appearing in (4.27), and take

$$
M=\delta^{2}
$$

By the above choice of $\delta, M$ and the hypotheses (4.25), we have that

$$
\begin{gathered}
k_{0} / 2-C_{\varphi}\left|\Delta \varphi^{n-1}\right|-C_{\varphi}\left|A \mathbf{u}^{n-1}\right|>k_{0} / 2-C_{\varphi} \delta-C_{\varphi} \delta \\
>k_{0} / 2-C_{\varphi} \cdot k_{0} /\left(8 C_{\varphi}\right)-C_{\varphi} \cdot k_{0} /\left(8 C_{\varphi}\right)=k_{0} / 4
\end{gathered}
$$

Similarly, we have

$$
\nu_{0} / 2-C_{u}\left|\Delta \varphi^{n-1}\right|-C_{u}\left|A \mathbf{u}^{n-1}\right|>\nu_{0} / 4
$$


Thus, from (4.20) and the last two inequalities, we get that

$$
\begin{aligned}
\frac{k_{0}}{4}\left|\Delta \varphi_{j}^{n}\right|^{2}+ & \frac{\nu_{0}}{4}\left|A \mathbf{u}_{j}^{n}\right|^{2}<\left(\frac{k_{0}}{2}-C_{\varphi}\left|\Delta \varphi^{n-1}\right|-C_{\varphi}\left|A \mathbf{u}^{n-1}\right|\right)\left|\Delta \varphi_{j}^{n}\right|^{2} \\
& +\left(\frac{\nu_{0}}{2}-C_{u}\left|\Delta \varphi^{n-1}\right|-C_{u}\left|A \mathbf{u}^{n-1}\right|\right)\left|A \mathbf{u}_{j}^{n}\right|^{2} \\
\leq & C_{0}\left(\left|\mathbf{u}_{j, t}^{n}\right|^{2}+\left|\varphi_{j, t}^{n}\right|^{2}+\left|\nabla \varphi_{j}^{n}\right|^{2}\right)+C_{0}\left(|\mathbf{h}|^{2}+|f|^{2}\right),
\end{aligned}
$$

where the positive constant $C_{0}$ depends on $|\mathbf{g}|_{L^{\infty} L^{4}}$, but not on $j$ or $n \in \mathbb{N}$.

Again by the above choice of $\delta, M$ and the hypotheses (4.25), we have that

$$
\frac{C_{1}}{2}<C_{1}-C_{2}\left(\left|\Delta \varphi^{n-1}\right|+\left|\nabla \mathbf{u}^{n-1}\right|+\left|\varphi_{t}^{n-1}\right|\right)
$$

By using these last two results in (4.28), we can write

$$
\begin{aligned}
& \Phi_{j}^{n}(t)+\frac{C_{1}}{2} \int_{0}^{t}\left|\Delta \varphi_{j}^{n}\right|^{2}+\left|A \mathbf{u}_{j}^{n}\right|^{2}+C_{1} \int_{0}^{t}\left(\left|\varphi_{j, t}^{n}\right|^{2}+\left|\mathbf{u}_{j, t}^{n}\right|^{2}+\left|\nabla \varphi_{j, t}^{n}\right|^{2}+\left|\nabla \mathbf{u}_{j, t}^{n}\right|^{2}\right) \\
& \leq C_{3} \Phi_{j}^{n}(0) \\
& +C_{4} \int_{0}^{t}\left(\left|\nabla \mathbf{u}_{t}^{n-1}\right|^{2}+\left|\nabla \varphi_{t}^{n-1}\right|^{2}\right)\left(|\mathbf{h}|^{2}+|f|^{2}\right) \\
& +C_{5} \int_{0}^{t}\left(\left|\nabla \mathbf{u}^{n-1}\right|+\left|\nabla \mathbf{u}_{t}^{n-1}\right|^{2}+\left|\nabla \varphi_{t}^{n-1}\right|^{2}+\alpha^{4}|\mathbf{g}|_{6}^{4}+\alpha^{2}|\mathbf{g}|_{3}^{2}+\alpha^{2}\left|\mathbf{g}_{t}\right|_{3}^{2}\right) \Phi_{j}^{n} \\
& +C_{2} \int_{0}^{t}\left(|f|^{2}+|\mathbf{h}|^{2}+\left|f_{t}\right|^{2}+\left|\mathbf{h}_{t}\right|^{2}\right),
\end{aligned}
$$

where

$$
\Phi_{j}^{n}(t)=\left|\nabla \mathbf{u}_{j}^{n}(t)\right|^{2}+\left|\nabla \varphi_{j}^{n}(t)\right|^{2}+\left|\mathbf{u}_{j, t}^{n}(t)\right|^{2}+\left|\varphi_{j, t}^{n}(t)\right|^{2} .
$$

By using Gronwall's lemma, we conclude in particular that

$$
\Phi_{j}^{n}(t)+C_{1} \int_{0}^{t}\left|\nabla \varphi_{j, t}^{n}\right|^{2}+\left|\nabla \mathbf{u}_{j, t}^{n}\right|^{2} \leq H_{1}(t) \exp H_{2}(t)
$$

where

$$
\begin{gathered}
H_{1}(t)=C_{3} \Phi_{j}^{n}(0)+C_{4} \int_{0}^{t}\left(\left|\nabla \mathbf{u}_{t}^{n-1}\right|^{2}+\left|\nabla \varphi_{t}^{n-1}\right|^{2}\right)\left(|\mathbf{h}|^{2}+|f|^{2}\right) \\
+C_{2} \int_{0}^{t}\left(|f|^{2}+|\mathbf{h}|^{2}+\left|f_{t}\right|^{2}+\left|\mathbf{h}_{t}\right|^{2}\right) \\
H_{2}(t)=C_{5} \int_{0}^{t}\left(\left|\nabla \mathbf{u}^{n-1}\right|+\left|\nabla \mathbf{u}_{t}^{n-1}\right|^{2}+\left|\nabla \varphi_{t}^{n-1}\right|^{2}+\alpha^{4}|\mathbf{g}|_{6}^{4}+\alpha^{2}|\mathbf{g}|_{3}^{2}+\alpha^{2}\left|\mathbf{g}_{t}\right|_{3}^{2}\right)
\end{gathered}
$$


Next, we take the initial data, the external fields $f$ and $g$ and $\alpha$ so small that (4.31)

$$
\left\{\begin{aligned}
(i) & \left\|\mathbf{u}_{0}||_{H_{0}^{1}}^{2}+\right\| \varphi_{0} \|_{H_{0}^{1}}^{2} \\
& +\left(\nu_{1}\left\|\mathbf{u}_{0}\right\|_{H_{0}^{1}}+\left\|\mathbf{u}_{0}\right\|_{H_{0}^{1}}|| \mathbf{u}_{0}\left\|_{H^{2}}+|\mathbf{h}|_{L^{\infty} L^{2}}+\alpha|\mathbf{g}|_{L^{\infty} L^{6}}+\right\| \varphi_{0} \|_{H_{0}^{1}}\right)^{2} \\
& +\left(k_{1}|| \varphi_{0}\left\|_{H_{0}^{1}}+\right\| \mathbf{u}_{0}\left\|_{H_{0}^{1}}\right\| \varphi_{0} \|_{H^{2}}+|f|_{L^{\infty} L^{2}}\right)^{2} \\
& <\frac{1}{20} \delta^{2} \min \left\{1, C_{1}, k_{0} / 4, \nu_{0} / 4,1 / C_{0}, 1 / C_{2}, 1 / C_{4}\right\} \\
(\text { ii }) & \sup _{0 \leq t \leq T}\left\{|f|^{2}+|\mathbf{h}|^{2}\right\}<\frac{1}{20} \delta^{2} \min \left\{1, C_{1}, k_{0} / 4, \nu_{0} / 4,1 / C_{0}, 1 / C_{2}, 1 / C_{4}\right\} \\
(\text { iii }) & \int_{0}^{T}\left(|f|^{2}+|\mathbf{h}|^{2}+\left|f_{t}\right|^{2}+\left|\mathbf{h}_{t}\right|^{2}\right) \\
& <\frac{1}{20} \delta^{2} \min \left\{1, C_{1}, k_{0} / 4, \nu_{0} / 4,1 / C_{0}, 1 / C_{2}, 1 / C_{4}\right\} \\
\text { (iv }) & C_{5} \int_{0}^{T} \alpha^{4}|\mathbf{g}|_{6}^{4}+\alpha^{2}|\mathbf{g}|_{3}^{2}+\alpha^{2}\left|\mathbf{g}_{t}\right|_{3}^{2} \leq 1
\end{aligned}\right.
$$

By using (4.1) and (4.2) at $t=0$ and the fact that the projections $P_{j}$ and $Q_{j}$ are respectively orthogonal projections on $V_{j}$ and $W_{j}$, we get

$$
\begin{aligned}
\Phi_{j}^{n}(0)= & \left|\nabla \mathbf{u}_{j}^{n}(0)\right|^{2}+\left|\nabla \varphi_{j}^{n}(0)\right|^{2}+\left|\mathbf{u}_{j, t}^{n}(0)\right|^{2}+\left|\varphi_{j, t}^{n}(0)\right|^{2} \\
& \leq\left\|\mathbf{u}_{0} \mid\right\|_{H_{0}^{1}}^{2}+\left\|\varphi_{0}\right\|_{H_{0}^{1}}^{2} \\
& +\left(\nu_{1}\left\|\mathbf{u}_{0}\right\|_{H_{0}^{1}}+\left\|\mathbf{u}_{0}\right\|_{H_{0}^{1}}\left\|\mathbf{u}_{0}\right\|_{H^{2}}+|\mathbf{h}|_{L^{\infty} L^{2}}+\alpha|\mathbf{g}|_{L^{\infty} L^{6}}+\left\|\varphi_{0}\right\|_{H_{0}^{1}}\right)^{2} \\
& +\left(k_{1}\left\|\varphi_{0}\right\|_{H_{0}^{1}}+\left\|\mathbf{u}_{0}\right\|_{H_{0}^{1}}\left\|\varphi_{0}\right\|_{H^{2}}+|f|_{L^{\infty} L^{2}}\right)^{2} \\
& <\frac{1}{20} \delta^{2} \min \left\{1, C_{1}, k_{0} / 4, \nu_{0} / 4,1 / C_{0}, 1 / C_{2}, 1 / C_{4}\right\}
\end{aligned}
$$

Therefore,

$$
\begin{aligned}
& \Phi_{j}^{n}(t)+C_{1} \int_{0}^{t}\left|\nabla \varphi_{j, t}^{n}\right|^{2}+\left|\nabla \mathbf{u}_{j, t}^{n}\right|^{2} \leq H_{1}(t) \exp H_{2}(t) \\
\leq & \frac{3 \delta^{2}}{20} \min \left\{1, C_{1}, k_{0} / 4, \nu_{0} / 4,1 / C_{0}, 1 / C_{2}, 1 / C_{4}\right\} e^{(2+T) \delta+1} \\
\leq & \frac{9}{20} \min \left\{1, C_{1}, k_{0} / 4, \nu_{0} / 4,1 / C_{0}, 1 / C_{2}, 1 / C_{4}\right\} \delta^{2}<\delta^{2},
\end{aligned}
$$

which gives four of the required estimates. The last two are obtained from the last result and (4.29) as follows:

$$
\begin{gathered}
\frac{k_{0}}{4}\left|\Delta \varphi_{j}^{n}\right|^{2}+\frac{\nu_{0}}{4}\left|A \mathbf{u}_{j}^{n}\right|^{2}<C_{0}\left(\left|\mathbf{u}_{j, t}^{n}\right|^{2}+\left|\varphi_{j, t}^{n}\right|^{2}+\left|\nabla \varphi_{j}^{n}\right|^{2}\right)+C_{0}\left(|\mathbf{h}|^{2}+|f|^{2}\right) \\
\leq\left(\frac{9}{20} \delta^{2}+\frac{1}{20} \delta^{2}\right) \min \left\{1, C_{1}, k_{0} / 4, \nu_{0} / 4,1 / C_{0}, 1 / C_{2}, 1 / C_{4}\right\},
\end{gathered}
$$

which gives the last two estimates.

Lemma 4.7. Fix $n \in \mathbb{N}$ and let $\varphi^{n-1}$ and $\mathbf{u}^{n-1}$ be given satisfying (4.25) with initial data, external fields, $\alpha$ and the constant $M$ as in Lemma 4.6 (these conditions are independent of $n \in \mathbb{N}$.) Then there are solutions $\varphi^{n}, \mathbf{u}^{n}$ respectively of problems (2.2) and (2.3). Moreover, such solutions satisfy

$$
\begin{array}{ll}
\sup _{0 \leq t \leq T}\left|\nabla \varphi^{n}\right|^{2}<M & \sup _{0 \leq t \leq T}\left|\nabla \mathbf{u}^{n}\right|^{2}<M \\
\sup _{0 \leq t \leq T}\left|\Delta \varphi^{n}\right|^{2}<M & \sup _{0 \leq t \leq T}\left|A \mathbf{u}^{n}\right|^{2}<M
\end{array}
$$




$$
\begin{array}{ll}
\sup _{0 \leq t \leq T}\left|\varphi_{t}^{n}\right|^{2}<M & \sup _{0 \leq t \leq T}\left|\mathbf{u}_{t}^{n}\right|^{2}<M \\
\int_{0}^{T}\left|\nabla \varphi_{t}^{n}\right|^{2}<M & \int_{0}^{T}\left|\nabla \mathbf{u}_{t}^{n}\right|^{2}<M .
\end{array}
$$

Proof. From the previous lemma, for each $j \in \mathbb{N}$, the solutions $\varphi_{j}^{n}$ and $\mathbf{u}_{j}^{n}$ of (4.1)(4.2) satisfies the estimate (4.26) uniformly with respect to $j$. Thus, in a standard way we can extract subsequences, which for simplicity we still denote $\left\{\varphi_{j}^{n}\right\}_{1}^{\infty}$ and $\left\{\mathbf{u}_{j}^{n}\right\}_{1}^{\infty}$, converging respectively to $\varphi^{n}, \mathbf{u}^{n}$, as $j \rightarrow+\infty$, in suitable topologies. Moreover, $\varphi^{n}, \mathbf{u}^{n}$ satisfy the same estimates as $\varphi_{j}^{n} \mathbf{u}_{j}^{n}$, respectively.

Since (4.1) and (4.2) are linear and $V_{j} \subset V_{j+1}, W_{j} \subset W_{j+1}$, as usual we can pass to the limit and show that $\varphi^{n}, \mathbf{u}^{n}$ satisfy the corresponding variational formulation of problems (2.2) and (2.3), respectively. Thus, we can find the corresponding pressure $p^{n}$ and due to the strong regularity of the obtained solutions get that $\varphi^{n}$, $\mathbf{u}^{n}$ satisfy respectively problems (2.2) and (2.3).

Now, we are ready to complete the proof of our first result.

\section{Proof of Theorem 2.1.}

We assume conditions (4.31) for the initial conditions, external fields and $\alpha$. The proof is done by finite induction.

For the basis of induction, we just observe that our iteration starts with $\varphi^{0}(t)=$ $\varphi_{0}$ and $\mathbf{u}^{0}(t)=u_{0}$. Then, being $M=\delta^{2}$ as in Lemma 4.6, we have:

$$
\begin{array}{ll}
\sup _{0 \leq t \leq T}\left|\partial_{t} \varphi^{0}\right|^{2}=0<M, & \sup _{0 \leq t \leq T}\left|\partial_{t} \mathbf{u}^{0}\right|^{2}=0<M, \\
\int_{0}^{T}\left|\nabla \partial_{t} \varphi^{0}\right|^{2}=0<M, & \int_{0}^{T}\left|\nabla \partial_{t} \mathbf{u}^{0}\right|^{2}=0<M .
\end{array}
$$

Besides, our choice of initial conditions also implies that

$$
\begin{array}{ll}
\sup _{0 \leq t \leq T}\left|\Delta \varphi^{0}\right|^{2}<M, & \sup _{0 \leq t \leq T}\left|A \mathbf{u}^{0}\right|^{2}<M, \\
\sup _{0 \leq t \leq T}\left|\nabla \varphi^{0}\right|^{2}<M, & \sup _{0 \leq t \leq T}\left|\nabla \mathbf{u}^{0}\right|^{2}<M .
\end{array}
$$

Next, the iteration part of the induction argument is just Lemma 4.7, and the theorem is proved.

\section{Proof of Theorem 2.2}

In order to prove Theorem 2.2, we need to estimate differences of two approximate solutions. For this, let $n, s \geq 1$ be any two natural numbers and denote

$$
\begin{aligned}
& \mathbf{u}^{n, s}(t)=\mathbf{u}^{n+s}(t)-\mathbf{u}^{n}(t), \\
& \varphi^{n, s}(t)=\varphi^{n+s}(t)-\varphi^{n}(t) .
\end{aligned}
$$

By subtracting the equations corresponding to the $n+s$-th and $n$-th iteration steps, we obtain the following equations for $\mathbf{u}^{n, s}$ and $\varphi^{n, s}$

$$
\begin{aligned}
& \mathbf{u}_{t}^{n, s}-\operatorname{div}\left(\nu\left(\varphi^{n+s-1}\right) \nabla \mathbf{u}^{n, s}\right)+\nabla p^{n, s}=\left(\mathbf{u}^{n-1, s} \cdot \nabla\right) \mathbf{u}^{\mathbf{n}}+\alpha \mathbf{g} \varphi^{n, s} \\
& +\operatorname{div}\left(\left(\nu\left(\varphi^{n+s-1}\right)-\nu\left(\varphi^{n-1}\right)\right) \nabla \mathbf{u}^{n}\right)-\left(\mathbf{u}^{n+s-1} \cdot \nabla\right) \mathbf{u}^{n, s},
\end{aligned}
$$




$$
\begin{aligned}
& \varphi_{t}^{n, s}-\operatorname{div}\left(k\left(\varphi^{n+s-1}\right) \nabla \varphi^{n, s}\right)=\left(\mathbf{u}^{n+s-1} \cdot \nabla\right) \varphi^{n, s} \\
& +\operatorname{div}\left(\left(k\left(\varphi^{n+s-1}\right)-k\left(\varphi^{n-1}\right)\right) \nabla \varphi^{n}\right)+\left(\mathbf{u}^{n-1, s} \cdot \nabla\right) \varphi^{n} .
\end{aligned}
$$

\section{Step 1: Some technical lemmas.}

Lemma 5.1. There exists a positive constants $0 \leq \delta \leq 1 / 2, C, \mu>0$, independent of $n$ and $s$, such that for each $n$ and $s \in \mathbb{N}$ :

$$
\begin{aligned}
& \frac{d}{d t}\left(\left|\nabla \mathbf{u}^{n, s}\right|^{2}+\left|\nabla \varphi^{n, s}\right|^{2}\right)+\mu\left(\left|A \mathbf{u}^{n, s}\right|^{2}+\left|\Delta \varphi^{n, s}\right|^{2}\right) \\
\leq & C\left(\left|\nabla \mathbf{u}^{n-1, s}\right|^{2}+\left|\nabla \varphi^{n-1, s}\right|^{2}\right)+C\left(\left|\nabla \mathbf{u}^{n, s}\right|^{2}+\left|\nabla \varphi^{n, s}\right|^{2}\right)+\delta\left|\Delta \varphi^{n-1, s}\right|^{2} .
\end{aligned}
$$

Proof. By multiplying (5.1) by $A \mathbf{u}^{n, s}$, we obtain

$$
\begin{aligned}
& \frac{1}{2} \frac{d}{d t}\left|\nabla \mathbf{u}^{n, s}\right|^{2}-\left(\operatorname{div}\left(\nu\left(\varphi^{n+s-1}\right) \nabla \mathbf{u}^{n, s}\right), A \mathbf{u}^{n, s}\right)=-\left(\mathbf{u}^{n-1, s} \cdot \nabla \mathbf{u}^{n}, A \mathbf{u}^{n, s}\right) \\
& +\left(\operatorname{div}\left(\left(\nu\left(\varphi^{n+s-1}\right)-\nu\left(\varphi^{n-1}\right)\right) \nabla \mathbf{u}^{n}, A \mathbf{u}^{n, s}\right)-\left(\mathbf{u}^{n+s-1} \cdot \nabla \mathbf{u}^{n, s}, A \mathbf{u}^{n, s}\right)\right. \\
& +\alpha\left(\mathbf{g} \varphi^{n, s}, A \mathbf{u}^{n, s}\right) .
\end{aligned}
$$

By using the identity $\operatorname{div}(\nu(\theta) \nabla \mathbf{v})=\nu(\theta) \Delta \mathbf{v}+\nu^{\prime}(\theta) \nabla(\theta) \nabla \mathbf{v}$ and Lemma 3.1, we then get

$$
\begin{gathered}
\frac{1}{2} \frac{d}{d t}\left|\nabla \mathbf{u}^{n, s}\right|^{2}+\left(\nu\left(\varphi^{n+s-1}\right) A \mathbf{u}^{n, s}, A \mathbf{u}^{n, s}\right)= \\
-\left(\left(\nu\left(\varphi^{n+s-1}\right)-\nu\left(\varphi^{n-1}\right)\right) A \mathbf{u}^{n}, A \mathbf{u}^{n, s}\right) \\
+\left(\left(\nu^{\prime}\left(\varphi^{n+s-1}\right) \nabla \varphi^{n+s-1}-\nu^{\prime}\left(\varphi^{n-1}\right) \nabla \varphi^{n-1}\right) \nabla \mathbf{u}^{n}, A \mathbf{u}^{n, s}\right) \\
+\left(\nu^{\prime}\left(\varphi^{n+s-1}\right) \nabla \varphi^{n+s-1} \nabla \mathbf{u}^{n, s}, A \mathbf{u}^{n, s}\right)-\left(\nu\left(\varphi^{n+s-1}\right) \nabla q^{n, s}, A \mathbf{u}^{n, s}\right) \\
\left(\left(\nu\left(\varphi^{n+s-1}\right)-\nu\left(\varphi^{n-1}\right)\right) \nabla q^{n}, A \mathbf{u}^{n, s}\right)-\left(\mathbf{u}^{n+s-1} \cdot \nabla \mathbf{u}^{n, s}, A \mathbf{u}^{n, s}\right) \\
-\left(\mathbf{u}^{n-1, s} \cdot \nabla \mathbf{u}^{n}, A \mathbf{u}^{n, s}\right)+\alpha\left(\mathbf{g} \varphi^{n, s}, A \mathbf{u}^{n, s}\right) .
\end{gathered}
$$

Next, we estimate the terms in the right-hand side by using Hölder's inequality, Sobolev embeddings and Young's inequality; we obtain

$$
\begin{aligned}
& \left|\left(\left(\nu\left(\varphi^{n+s-1}\right)-\nu\left(\varphi^{n-1}\right)\right) A \mathbf{u}^{n}, A \mathbf{u}^{n, s}\right)\right| \\
& \leq C\left|\nu\left(\varphi^{n+s-1}\right)-\nu\left(\varphi^{n-1}\right)\right|_{L^{\infty}}\left|A \mathbf{u}^{n}\right|\left|A \mathbf{u}^{n, s}\right| \\
& \leq C\left|\varphi^{n-1, s}\right|_{L^{\infty}}\left|A \mathbf{u}^{n}\right|\left|A \mathbf{u}^{n, s}\right| \\
& \leq C\left|\nabla \varphi^{n-1, s}\right|^{1 / 2}\left|\Delta \varphi^{n-1, s}\right|^{1 / 2}\left|A \mathbf{u}^{n, s}\right| \leq C_{\varepsilon}\left|\nabla \varphi^{n-1, s}\right|\left|\Delta \varphi^{n-1, s}\right|+\varepsilon\left|A \mathbf{u}^{n, s}\right|^{2} \\
& \leq C_{\varepsilon, \varepsilon_{1}}\left|\nabla \varphi^{n-1, s}\right|^{2}+\varepsilon_{1}\left|\Delta \varphi^{n-1, s}\right|^{2}+\varepsilon\left|A \mathbf{u}^{n-1, s}\right|^{2} \text {, } \\
& \left|\left(\left(\nu^{\prime}\left(\varphi^{n+s-1}\right) \nabla \varphi^{n+s-1}-\nu^{\prime}\left(\varphi^{n-1}\right) \nabla \varphi^{n-1}\right) \nabla \mathbf{u}^{n}, A \mathbf{u}^{n, s}\right)\right| \\
& \leq C \mid \nu^{\prime}\left(\varphi^{n+s-1}\right) \nabla \varphi^{n-1, s}+\left(\left(\nu^{\prime}\left(\varphi^{n+s-1}\right)-\left.\nu^{\prime}\left(\varphi^{n-1}\right) \nabla \varphi^{n-1}\right|_{3}\left|\nabla \mathbf{u}^{n}\right|_{6}\left|A \mathbf{u}^{n, s}\right|\right.\right. \\
& \leq C\left(\nu_{1}^{\prime}\left|\nabla \varphi^{n-1, s}\right|_{3}+\left|\varphi^{n-1, s}\right|_{6}\left|\nabla \varphi^{n-1}\right|_{6}\right)\left|A \mathbf{u}^{n, s}\right| \\
& \leq C_{\varepsilon, \varepsilon_{1}}\left|\nabla \varphi^{n-1, s}\right|^{2}+\varepsilon_{1}\left|\Delta \varphi^{n-1, s}\right|^{2}+C_{\varepsilon}\left|\nabla \varphi^{n-1, s}\right|^{2}+\varepsilon\left|A \mathbf{u}^{n, s}\right|^{2}, \\
& \left|\left(\nu^{\prime}\left(\varphi^{n+s-1}\right) \nabla \varphi^{n+s-1} \nabla \mathbf{u}^{n, s}, A \mathbf{u}^{n, s}\right)\right| \leq C \nu_{1}^{\prime}\left|\nabla \varphi^{n+s-1}\right|_{4}\left|\nabla \mathbf{u}^{n, s}\right|_{4}\left|A \mathbf{u}^{n, s}\right| \\
& \leq C\left|\Delta \varphi^{n+s-1}\right|\left|\nabla \mathbf{u}^{n, s}\right|^{1 / 4}\left|A \mathbf{u}^{n, s}\right|^{7 / 4} \\
& \leq C_{\varepsilon}\left|\nabla \mathbf{u}^{n, s}\right|^{2}+\varepsilon\left|A \mathbf{u}^{n, s}\right|^{2} \\
& \left|\left(\nu\left(\varphi^{n+s-1}\right) \nabla q^{n, s}, A \mathbf{u}^{n, s}\right)\right|=\left|\left(q^{n, s}, \operatorname{div}\left(\nu\left(\varphi^{n+s-1}\right) A \mathbf{u}^{n, s}\right)\right)\right| \\
& =\left|\left(q^{n, s}, \nu^{\prime}\left(\varphi^{n+s-1}\right) \nabla \varphi^{n+s-1} A \mathbf{u}^{n, s}\right)\right| \\
& \leq C \nu_{1}^{\prime}\left|q^{n, s}\right|_{4}\left|\nabla \varphi^{n+s-1}\right|_{4}\left|A \mathbf{u}^{n, s}\right| \\
& \leq C\left|q^{n, s}\right|^{1 / 4}\left|\left\|q^{n, s}\right\|_{1}^{3 / 4}\right| \Delta \varphi^{n+s-1}|| A \mathbf{u}^{n, s} \mid \\
& \leq C_{\varepsilon}\left|\nabla \mathbf{u}^{n, s}\right|^{2}+\varepsilon\left|A \mathbf{u}^{n, s}\right|^{2} \text {, }
\end{aligned}
$$




$$
\begin{aligned}
& \mid\left(\left(\nu\left(\varphi^{n+s-1}\right)-\nu\left(\varphi^{n-1}\right)\right) \nabla q^{n},\right.\left.A \mathbf{u}^{n, s}\right)\left.|\leq C| \varphi^{n-1, s}\right|_{\infty}\left|\nabla q^{n}\right|\left|A \mathbf{u}^{n, s}\right| \\
& \leq C\left|\nabla \varphi^{n-1, s}\right|^{1 / 2}\left|\Delta \varphi^{n-1, s}\right|^{1 / 2}\left|A \mathbf{u}^{n}\right|\left|A \mathbf{u}^{n, s}\right| \\
& \leq C_{\varepsilon, \varepsilon_{1}}\left|\nabla \varphi^{n-1, s}\right|^{2}+\varepsilon_{1}\left|\Delta \varphi^{n-1, s}\right|^{2}+\varepsilon\left|A \mathbf{u}^{n, s}\right|^{2} \\
&\left|\left(\mathbf{u}^{n+s-1} \cdot \nabla \mathbf{u}^{n, s}, A \mathbf{u}^{n, s}\right)\right| \leq C\left|\mathbf{u}^{n+s-1}\right|_{L^{6}}\left|\nabla \mathbf{u}^{n, s}\right|_{L^{3}}\left|A \mathbf{u}^{n, s}\right| \\
& \leq C\left|\nabla \mathbf{u}^{n+s-1}\right|\left|\nabla \mathbf{u}^{n, s}\right|^{1 / 2}\left|A \mathbf{u}^{n, s}\right|^{3 / 2} \\
& \leq C_{\varepsilon}\left|\nabla \mathbf{u}^{n, s}\right|^{2}+\varepsilon\left|A \mathbf{u}^{n, s}\right|^{2}, \\
&\left|\left(\mathbf{u}^{n-1, s} \cdot \nabla \mathbf{u}^{n}, A \mathbf{u}^{n, s}\right)\right| \leq C\left|\mathbf{u}^{n-1, s}\right|_{L^{6}}\left|\nabla \mathbf{u}^{n}\right|_{L^{3}}\left|A \mathbf{u}^{n, s}\right| \\
& \leq C\left|\nabla \mathbf{u}^{n-1, s}\right|\left|A \mathbf{u}^{n}\right|\left|A \mathbf{u}^{n, s}\right| \\
& \leq C_{\varepsilon}\left|\nabla \mathbf{u}^{n-1, s}\right|^{2}+\varepsilon\left|A \mathbf{u}^{n, s}\right|^{2} \\
& \\
&\left|\alpha\left(\mathbf{g} \varphi^{n, s}, A \mathbf{u}^{n, s}\right)\right| \leq C|\mathbf{g}|_{L^{3}}\left|\varphi^{n, s}\right|_{L^{6}}\left|A \mathbf{u}^{n, s}\right| \\
& \leq C_{\varepsilon}\left|\nabla \varphi^{n, s}\right|^{2}+\varepsilon\left|A \mathbf{u}^{n, s}\right|^{2}
\end{aligned}
$$

By taking $\varepsilon>0, \varepsilon_{1}>0$ sufficiently small in the previous estimates, we obtain in (5.4) the following differential inequality with a constant $0 \leq \delta_{1} \leq 1 / 4$ :

$$
\begin{gathered}
\frac{d}{d t}\left|\nabla \mathbf{u}^{n, s}(t)\right|^{2}+\nu_{0}\left|A \mathbf{u}^{n, s}\right|^{2} \\
\leq C\left(\left|\nabla \mathbf{u}^{n-1, s}\right|^{2}+\left|\nabla \mathbf{u}^{n, s}\right|^{2}\right)+C\left|\nabla \varphi^{n-1, s}\right|^{2}+\delta_{1}\left|\Delta \varphi^{n-1, s}\right|^{2} .
\end{gathered}
$$

Analogously, by multiplying (5.2) by $-\Delta \varphi^{n, s}$, we obtain the following inequality with a constant $0 \leq \delta_{2} \leq 1 / 4$ :

$$
\begin{gathered}
\frac{1}{2} \frac{d}{d t}\left|\nabla \varphi^{n, s}\right|^{2}+k_{0}\left|\Delta \varphi^{n, s}\right|^{2} \\
\leq C\left(\left|\nabla \mathbf{u}^{n-1, s}\right|^{2}+\left|\nabla \varphi^{n-1, s}\right|^{2}+\left|\nabla \varphi^{n, s}\right|^{2}\right)+\delta_{2}\left|\Delta \varphi^{n-1, s}\right|^{2} .
\end{gathered}
$$

By adding (5.4) and (5.5) and calling $\delta=\delta_{1}+\delta_{2}$, we obtain (5.3).

Lemma 5.2. There exists a positive constant $C>0$, independent of $n$ and $s$, such that for each $n$ and $s \in \mathbb{N}$ we have:

$$
\begin{aligned}
\left|\mathbf{u}_{t}^{n, s}\right|^{2}+\left|\varphi_{t}^{n, s}\right|^{2} \leq & C\left(\left|A \mathbf{u}^{n, s}\right|^{2}+\left|\Delta \varphi^{n, s}\right|^{2}+\left|\nabla \varphi^{n-1, s}\right|^{2}\right. \\
& \left.+\left|\nabla \mathbf{u}^{n-1, s}\right|^{2}+\left|\nabla \varphi^{n-1, s}\right|^{2}\right) .
\end{aligned}
$$

Proof. By considering $\mathbf{u}_{t}^{n, s}$ as a test function in (5.1), we obtain

$$
\begin{gathered}
\left|\mathbf{u}_{t}^{n, s}\right|^{2}=\left(\operatorname{div}\left(\nu\left(\varphi^{n+s-1}\right) \nabla \mathbf{u}^{n, s}\right), \mathbf{u}_{t}^{n, s}\right)+\left(\left(\mathbf{u}^{n-1, s} \cdot \nabla\right) \mathbf{u}^{\mathbf{n}}, \mathbf{u}_{t}^{n, s}\right) \\
+\alpha\left(\mathbf{g} \varphi^{n, s}, \mathbf{u}_{t}^{n, s}\right)+\left(\operatorname{div}\left(\left(\nu\left(\varphi^{n+s-1}\right)-\nu\left(\varphi^{n-1}\right)\right) \nabla \mathbf{u}^{n}\right), \mathbf{u}_{t}^{n, s}\right) \\
-\left(\left(\mathbf{u}^{n+s-1} \cdot \nabla\right) \mathbf{u}^{n, s}, \mathbf{u}_{t}^{n, s}\right) .
\end{gathered}
$$

Next, by considering $\varphi_{t}^{n, s}$ as a test function in (5.2), we find

$$
\begin{aligned}
& \left|\varphi_{t}^{n, s}\right|^{2}=\left(\operatorname{div}\left(k\left(\varphi^{n+s-1}\right) \nabla \varphi^{n, s}\right), \varphi_{t}^{n, s}\right)+\left(\left(\mathbf{u}^{n+s-1} \cdot \nabla\right) \varphi^{n, s}, \varphi_{t}^{n, s}\right) \\
& +\left(\operatorname{div}\left(\left(k\left(\varphi^{n+s-1}\right)-k\left(\varphi^{n-1}\right)\right) \nabla \varphi^{n}\right), \varphi_{t}^{n, s}\right)+\left(\left(\mathbf{u}^{n-1, s} \cdot \nabla\right) \varphi^{n}, \varphi_{t}^{n, s}\right) .
\end{aligned}
$$

By estimating the right-hand side of (5.6) and (5.7) as before, using the fact that that from Theorem 2.1, $\left\|\Delta \varphi^{n-1, s}\right\|_{L^{\infty}\left(0, T ; L^{2}(\Omega)\right)} \leq\left\|\Delta \varphi^{n-1}\right\|_{L^{\infty}\left(0, T ; L^{2}(\Omega)\right)}+$ $\left\|\Delta \varphi^{s}\right\|_{L^{\infty}\left(0, T ; L^{2}(\Omega)\right)} \leq 2 M$, independently of $s$ and $n$, we get (5.6). 
Lemma 5.3. There exists a positive constant $C>0$, independent of $n$ and $s$, such that for each $n$ and $s \in \mathbb{N}$ we have:

$$
\begin{gathered}
\frac{d}{d t}\left(\left|\nabla \mathbf{u}^{n, s}\right|^{2}+\left|\nabla \varphi^{n, s}\right|^{2}\right)+\left|A \mathbf{u}^{n, s}\right|^{2}+\left|\Delta \varphi^{n, s}\right|^{2} \leq \\
C\left(\left|\nabla \mathbf{u}^{n, s}\right|^{2}+\left|\nabla \varphi^{n, s}\right|^{2}+\left|\nabla \mathbf{u}^{n-1, s}\right|^{2}+\left|\nabla \varphi^{n-1, s}\right|^{2}+\left|\nabla \varphi^{n-1, s}\right|\right) .
\end{gathered}
$$

Proof. By multiplying again (5.1) by $A \mathbf{u}^{n, s}$ and (5.2) by $\Delta \varphi^{n, s}$, using as before that by theorem 2.1, $\left\|\Delta \varphi^{n-1, s}\right\|_{L^{\infty}\left(0, T ; L^{2}(\Omega)\right)} \leq 2 M$, independently of $s$ and $n$, we easily obtain (5.7).

Step 2: Passage to the limit. From (5.3), by setting $a_{n}(t)=\left|\nabla \mathbf{u}^{n, s}(t)\right|^{2}+$ $\left|\nabla \varphi^{n, s}(t)\right|^{2}, b_{n}(t)=\mu\left(\left|A \mathbf{u}^{n, s}(t)\right|^{2}+\left|\Delta \varphi^{n, s}(t)\right|^{2}\right), c_{n}(t)=C$ and $d_{n}(t)=C$, and using lemma 3.3 and recalling that $a_{n}(0)=0$, we conclude in particular that the sequence $a_{n}(t)$ and $\int_{0}^{t} b_{n}(s) d s$ are Cauchy, uniformly with respect to $t \in[0, T]$.

Thus, our sequences of approximate solutions are Cauchy sequences (in suitable Banach spaces,) and, by using also (5.6), we conclude that there are $\mathbf{u}$ and $\varphi$ such that as $n \rightarrow \infty$ :

$\mathbf{u}^{n} \rightarrow \mathbf{u}$ strongly in $L^{\infty}(V) \cap L^{2}(D(A))$,

$\mathbf{u}_{t}^{n} \rightarrow \mathbf{u}_{t}$ strongly in $L^{2}(H)$,

$\varphi^{n} \rightarrow \varphi$ strongly in $L^{\infty}\left(H_{0}^{1}\right) \cap L^{2}\left(H^{2}\right)$,

$\varphi_{t}^{n} \rightarrow \varphi_{t}$ strongly in $L^{2}\left(L^{2}\right)$.

With the help of these convergences, it is then a standard procedure to pass to the limit in the approximate equations to obtain the following.

$$
\begin{aligned}
& \int_{0}^{t}\left\langle\mathbf{u}_{t}-\operatorname{div}(\nu(\varphi) \nabla \mathbf{u})+\mathbf{u} \cdot \nabla \mathbf{u}-\alpha \mathbf{g} \varphi-\mathbf{h}, v\right\rangle \phi(t) d t=0, \\
& \int_{0}^{t}\left\langle\varphi_{t}-\operatorname{div}(k(\varphi) \nabla \varphi)+\mathbf{u} \cdot \nabla \varphi-f, \psi\right\rangle \beta(t) d t=0,
\end{aligned}
$$

for all $\mathbf{v} \in L^{2}(\Omega), \psi \in L^{2}(\Omega)$ and $\phi, \beta \in L^{\infty}(0, T)$, which imply (2.4). The verification that the initial conditions are satisfied is also a standard procedure. Thus, $(\mathbf{u}, \varphi)$ is a strong solution (see the next section for information concerning the associated pressure,) and as usual, all the previously obtained estimates hold for them. With these estimates, it then again standard to prove the uniqueness of such solutions.

Step 3: Rates of convergence. The required convergence-rate bounds can be obtained as follows. Let

$$
\begin{gathered}
\mathbf{v}^{n}=\mathbf{u}^{n}-\mathbf{u} \\
z^{n}=\varphi^{n}-\varphi \\
q^{n}=p^{n}-p
\end{gathered}
$$

Next, by subtracting the corresponding equations for the velocity, using then $\mathbf{v}^{\mathbf{n}}$ as a multiplier and proceeding in a standard way with the help of our hypotheses on $\nu$, after some computations we get:

$$
\begin{aligned}
\frac{1}{2} \frac{d}{d t}\left|\mathbf{v}^{n}\right|^{2}+\nu_{0}\left|\nabla \mathbf{v}^{n}\right|^{2} \\
\\
\quad \leq \nu_{1}^{\prime}\left|z^{n-1}\right|{ }_{4}\left|\nabla \mathbf{u}^{n}\right|_{4}\left|\nabla \mathbf{v}^{n}\right|+\left|\mathbf{v}^{n-1}\right|\left|\nabla \mathbf{u}^{n}\right|{ }_{4}\left|\mathbf{v}^{n}\right|_{4}+\alpha|\mathbf{g}|_{6}\left|z^{n}\right|\left|\mathbf{v}^{n}\right|_{3} \\
\quad \leq C_{\epsilon}\left|\nabla z^{n-1}\right|^{2}+\epsilon\left|\nabla \mathbf{v}^{n}\right|^{2}+C_{\epsilon}\left|\mathbf{v}^{n-1}\right|^{2}+\epsilon\left|\nabla \mathbf{v}^{n}\right|^{2}+C\left|z^{n}\right|^{2}+\epsilon\left|\nabla \mathbf{v}^{n}\right|^{2},
\end{aligned}
$$


for any $\epsilon>0$ and suitable positive constants $C_{\epsilon}$ and $C$ independent of $n$ and $t \in[0, T]$; here we used Sobolev embeddings and the fact that $\left|\Delta \mathbf{u}^{n}\right|$ is uniformly bounded in time.

By taking $\epsilon=\nu_{0} / 6$ in this inequality, we are left with

$$
\frac{d}{d t}\left|\mathbf{v}^{n}\right|^{2}+\nu_{0}\left|\nabla \mathbf{v}^{n}\right|^{2} \leq C_{1}\left|\nabla z^{n-1}\right|^{2}+C_{2}\left|\mathbf{v}^{n-1}\right|^{2}+C_{3}\left|\nabla z^{n}\right|^{2},
$$

By subtracting the equations for $\varphi^{n}$ and $\varphi$, using $z^{n}$ as a multiplier, proceeding in standard way, after some computations, we get:

$$
\begin{array}{r}
\frac{1}{2} \frac{d}{d t}\left|z^{n}\right|^{2}+k_{0}\left|\nabla z^{n}\right|^{2} \leq C\left|z^{n-1}\right|_{4}\left|\nabla \varphi^{n}\right|_{4}\left|\nabla z^{n}\right|+\left|\mathbf{v}^{n-1}\right|\left|\nabla \varphi^{n}\right|_{4}\left|z^{n}\right|_{4} \\
\leq C_{\epsilon}\left|\nabla z^{n-1}\right|^{2}+\epsilon\left|\nabla z^{n}\right|^{2}+C_{\epsilon}\left|\mathbf{v}^{n-1}\right|^{2}+\epsilon\left|\nabla z^{n}\right|^{2},
\end{array}
$$

as before, for any $\epsilon>0$ and suitable positive constants $C_{\epsilon}$ and $C$ independent of $n$ and $t \in[0, T]$; here we used Sobolev embeddings and the fact that $\left|\Delta \varphi^{n}\right|$ is uniformly bounded in time and with respect to $n$.

By taking $\epsilon=k_{0} / 4$ in this inequality, we are left with

$$
\frac{d}{d t}\left|z^{n}\right|^{2}+k_{0}\left|\nabla z^{n}\right|^{2} \leq C_{4}\left|\nabla z^{n-1}\right|^{2}+C_{5}\left|\mathbf{v}^{n-1}\right|^{2} .
$$

By multiplying (5.8) by $C_{6}=2 C_{3} / k_{0}$ and adding to (5.7), we have:

$$
\begin{aligned}
& \frac{d}{d t}\left(\left|\mathbf{v}^{n}\right|^{2}+C_{6}\left|z^{n}\right|^{2}\right)+\nu_{0}\left|\nabla \mathbf{v}^{n}\right|^{2}+\left(C_{3} / k_{0}\right)\left|\nabla z^{n}\right|^{2} \\
& \leq\left(C_{2}+C_{5} C_{6}\right)\left|\mathbf{v}^{n-1}\right|^{2}+\left(C_{1}+C_{4} C_{6}\right)\left|\nabla z^{n-1}\right|^{2} \\
& \leq\left(C_{2}+C_{5} C_{6}\right)\left(\left|\mathbf{v}^{n-1}\right|^{2}+C_{6}\left|z^{n-1}\right|^{2}\right) \\
& +\left(C_{1}+C_{4} C_{6}\right)\left(k_{0} / C_{3}\right)\left(\nu_{0}\left|\nabla \mathbf{v}^{n-1}\right|^{2}+\left(C_{3} / k_{0}\right)\left|\nabla z^{n-1}\right|^{2}\right)
\end{aligned}
$$

By calling $a_{n}(t)=\left|\mathbf{v}^{n}\right|^{2}(t)+C_{6}\left|z^{n}\right|^{2}(t), b_{n}(t)=\nu_{0}\left|\nabla \mathbf{v}^{n}\right|^{2}(t)+\left(C_{3} / k_{0}\right)\left|\nabla z^{n}\right|^{2}(t)$, observing that $a_{n}(0)=0$, for all $n$, and using lemma 3.2, we conclude that are positive constants $C$ and $D$, independent of $n$ and $t \in[0, T]$ such that

$$
\left|\mathbf{v}^{n}\right|^{2}(t)+C_{6}\left|z^{n}\right|^{2}(t)+\int_{0}^{t}\left(\nu_{0}\left|\nabla \mathbf{v}^{n}\right|^{2}(s)+\left(C_{3} / k_{0}\right)\left|\nabla z^{n}\right|^{2}(s)\right) d s \leq C \frac{(D t)^{n}}{n !},
$$

from which follows (2.5) and (2.6). Then, by using this last result and the estimates of lemmas 5.2 and 5.3 , integrated in time, we obtain (2.7), (2.8) and (2.9).

\section{Results on the Pressure}

By using the Amrouche and Girault [1] results on the Stokes problem and the estimates given in the above sections, we easily obtain the following propositions.

Proposition 6.1. Under the hypotheses of Theorem 2.1 for each $n \in \mathbb{N}$, there exists $p^{n} \in L^{\infty}\left(0, T ; H^{1}(\Omega) / \mathbb{R}\right)$ such that

$$
\sup _{t}\left\{\left\|p^{n}(t)\right\|_{H^{1}(\Omega) / \mathbb{R}}^{2}\right\} \leq C_{0},
$$

for all $t \in[0, T]$, where $C_{0}$ is a positive constant independent of $n$. 
Proposition 6.2. Under the hypotheses of Theorem 2.2, the approximate pressures, $p^{n}$, converge in the space $L^{\infty}\left(0, T ; H^{1}(\Omega) / \mathbb{R}\right)$ to a limit $p$ such that $(\mathbf{u}, \varphi, p)$ is a solution of problem (1)-(2); moreover such solution is unique ( $p$ up to a constant.) Also, for each $n \in \mathbb{N}$, the approximate pressure satisfies the following rate of convergence:

$$
\int_{0}^{t}\left|p^{n}(\tau)-p(\tau)\right|_{H^{1}(\Omega) / \mathbb{R}}^{2} d \tau \leq C\left(\frac{(D t)^{n}}{n !}+\frac{(D t)^{n-1}}{(n-1) !}+\frac{(D t)^{(n-1) / 2}}{((n-1) / 2) !}\right)
$$

for all $t \in[0, T]$, with positive constants independent of $n$.

Proof. We observe that the equation satisfied by $p^{n, s}$ is

$$
\begin{aligned}
& \mathbf{u}_{t}^{n, s}-\operatorname{div}\left(\nu\left(\varphi^{n+s}\right) \nabla \mathbf{u}^{n, s}\right)+\nabla p^{n, s}=\left(\mathbf{u}^{n-1, s} \cdot \nabla\right) \mathbf{u}^{\mathbf{n}}+\alpha \mathbf{g} \varphi^{n, s} \\
& +\operatorname{div}\left(\left(\nu\left(\varphi^{n+s}\right)-\nu\left(\varphi^{n}\right)\right) \nabla \mathbf{u}^{n}\right)-\left(\mathbf{u}^{n+s-1} \cdot \nabla\right) \mathbf{u}^{n, s} .
\end{aligned}
$$

Consequently

$$
\begin{aligned}
& \left|\nabla p^{n, s}\right|^{2} \leq C\left[\left|\mathbf{u}_{t}^{n, s}\right|^{2}+\left|\operatorname{div}\left(\nu\left(\varphi^{n+s}\right) \nabla \mathbf{u}^{n, s}\right)\right|^{2}+\left|\left(\mathbf{u}^{n-1, s} \cdot \nabla\right) \mathbf{u}^{\mathbf{n}}\right|^{2}\right. \\
& +\left|\left(\mathbf{u}^{n+s-1} \cdot \nabla\right) \mathbf{u}^{n, s}\right|^{2} \leq C\left[\left|\mathbf{u}_{t}^{n, s}\right|^{2}+\left|A \mathbf{u}^{n, s}\right|^{2}+\left|\nabla \mathbf{u}^{n-1, s}\right|^{2}+\left|\nabla \mathbf{u}^{n, s}\right|^{2}\right] .
\end{aligned}
$$

This inequality together with our previous estimates imply the stated result.

Acknowledgment: The authors are indebted to F. Guillén-González for several fruitful discussions.

\section{REFERENCES}

[1] C. Amrouche, and V. Girault, On the existence and regularity of the solutions of Stokes Problem an arbitrary dimension, Proc. Japan Acad. Sect. A, 67 (1991), 171-175.

[2] S. Chandrasekhar, Hydrodynamic and hydromagnetic stability, Dover, New York, 1981.

[3] J.I. Diaz, G. Galiano, On the Boussinesq system with non linear thermal diffusion, Nonlinear Analysis, Theory, Methods \& Applications, 30, No. 6 (1997), 3255-3263.

[4] P.G. Drazin, W. H. Reid, Hydrodynamic Stability, Cambridge University Press, 1981.

[5] B. Climent-Ezquerra, F. Guillén-González, M.A. Rojas-Medar, Time-periodic solutions for a generalized Boussinesq model with Neumann boundary conditions for temperature, Proc. $R$. Soc. A, 463 (2007), 2153-2164.

[6] E. Feireisl, Dynamics of Viscous Incompressible Fluids, Oxford University Press, Oxford, 2004.

[7] E. Feireisl, J. Málek, On the Navier-Stokes equations with temperature dependent transport coefficients, Differ. Equ. Nonlinear Mech. Art. ID 90616, (2006), 14 pp. (electronic).

[8] G.P. Galdi, An Introduction to the Mathematical Theory of the Navier-Stokes Equations, Vol. I, Springer-Verlag, New York, 1994.

[9] F. Guillén-González, P. Damázio, M.A. Rojas-Medar, Approximation by an iterative method for regular solutions for incompressible fluids with mass diffusion, J. Math. Anal. Appl., 326 (2007), 468-487.

[10] F. Guillén-González, Mamadou Sy, Iterative method for mass diffusion model with density dependent viscosity, Submitted for publication.

[11] F. Guillén-González, M.A. Rodriguez-Bellido, Convergence and error estimate of two iterative methods for the strong solution of the Korteweg model, Submitted for publication.

[12] T. Hishida, Existence and regularizing properties of solutions for the nonstationary convection problem, Funkcialy Ekvaciy, 34 (1991), 449-474.

[13] Y. Kagei, On weak solutions of nonstationary Boussinesq equations, Differential Integral Equations, 6 (1993), no. 3, 587-611.

[14] Y. Kagei, W. von Wahl, Stability of higher norms in terms of energy-stability for the Boussinesq equations: remarks on the asymptotic behaviour of convection-roll-type solutions, Differential Integral Equations, 7 (1994), no. 3-4, 921-948.

[15] D. D. Joseph, Stability of Fluid Motion, Springer-Verlag, Berlin, 1976. 
[16] J.L. Lions, Quèlques mèthods de rèsolution des problèmes aux limits non linèares, Dunod, Paris, 1969.

[17] S.A. Lorca, J.L. Boldrini, Stationary solutions for generalized Boussinesq models, J. Diferential Equations, 124 (1996), 389-406.

[18] S.A. Lorca, J.L. Boldrini, The initial value problem for a generalized Boussinesq model, Nonlinear Analysis, 36 (1999), 457-480.

[19] S.A. Lorca, J.L. Boldrini, The initial value problem for a generalized Boussinesq model: regularity and global existence of strong solutions, Mat. Contemp., 11 (1996), 71-94.

[20] J. Málek, M. Ruzicka, G. Thäter, Fractal dimension, attractors, and the Boussinesq approximation in three dimensions, Mathematical problems for Navier-Stokes equations (Centro, 1993). Acta Appl. Math., 37 (1994), no. 1-2, 83-97

[21] A.C. Moretti, M.A. Rojas-Medar, M.D. Rojas Medar, Reproductive weak solutions for generalized Boussinesq models in exterior domains, Mat. Contemp., 23 (2002), 119-137.

[22] A.C. Moretti, M.A. Rojas-Medar, M. Drina Rojas-Medar, The equations of a viscous incompressible chemically active fluid: existence and uniqueness of strong solutions in an unbounded domain, Comput. Math. Appl., 44 (2002), 287-299.

[23] H. Morimoto, Nonstationary Boussinesq equations, J. Fac. Sci., Univ Tokyo, Sect., IA Math., 39 (1992), 61-75.

[24] E.A. Notte-Cuello, M.A. Rojas-Medar Stationary solutions for generalized Boussinesq models in exterior domains, Electron. J. Differential Equations, No. 22, (1998).

[25] K.R. Rajagopal, M. Ruzicka, A.R. Srinivasa, On the Oberbeck-Boussinesq approximation, Math. Models Methods Appl. Sci., 6 (1996), no. 8, 1157-1167.

[26] M.A Rojas-Medar, S.A. Lorca, The equation of a viscous incompressible chemical active fluid I: uniqueness and existence of the local solutions, Rev. Mat. Apl. (1995), 16, 57-80.

[27] M.A Rojas-Medar, S.A. Lorca, Global strong solution of the equations for the motion of a chemical active fluid, Mat. Contemp., 8 (1995), 319-335.

[28] M.A Rojas-Medar, S.A. Lorca, An error estimate uniform in time for spectral Galerkin approximations for the equations for the motion of chemical active fluid, Rev. Univ. Complutense de Madrid, 18 (1995), 431-458.

[29] H. Sohr, The Navier-Stokes Equatios. An Elementary Functional Analytic Approach, Birkhäuser Verlag, Basel, 2001.

[30] R. Temam, Navier-Stokes equations, North-Holland, Amsterdan (1977).

[31] H. von Tippelkirch, Über Konvektionszeller insbesondere in flüssigen Schefel, Beiträge Phys. Atmos., 20 (1956), 37-54.

IMECC-UNICAMP, CP 6065, 13083-859, CAMPINAS-SP, BRAZIL

E-mail address: boldrini@ime.unicamp.br

Dpto. de Ecuaciones Diferenciales y Análisis Numérico, Facultad de Matemáticas, Universidad de Sevilla, Sevilla, 41012, Spain

E-mail address: bcliment@us.es

Dpto. de Matemática, Universidad de Antofagasta, Casilla 170, Antofagasta, Chile E-mail address: mrojas@uantof.cl

Dpto. de Ciencias Básicas, Facultad de Ciencias, Universidad del Bío-Bío, Campus Fernando May, Casilla 447, Chillán, Chile

E-mail address: marko@ueubiobio.cl 